\title{
Investigation of the leading and subleading high-energy behavior of hadron-hadron total cross sections using a best-fit analysis of hadronic scattering data
}

\author{
M. Giordano, ${ }^{1, *}$ E. Meggiolaro, ${ }^{2, \dagger}$ and P. V. R. G. Silva ${ }^{3,+}$ \\ ${ }^{1}$ Institute for Theoretical Physics, Eötvös University, and MTA-ELTE "Lendület" Lattice Gauge Theory \\ Research Group, Pázmány P. sétány 1/A, H-1117 Budapest, Hungary \\ ${ }^{2}$ Dipartimento di Fisica dell'Università di Pisa, and INFN, Sezione di Pisa, \\ Largo Pontecorvo 3, I-56127 Pisa, Italy \\ ${ }^{3}$ Instituto de Física Gleb Wataghin, Universidade Estadual de Campinas-UNICAMP, \\ Campinas - São Paulo 13083-859, Brazil
}

(Received 6 March 2017; published 21 August 2017)

\begin{abstract}
In the present investigation we study the leading and subleading high-energy behavior of hadron-hadron total cross sections using a best-fit analysis of hadronic scattering data. The parametrization used for the hadron-hadron total cross sections at high energy is inspired by recent results obtained by Giordano and Meggiolaro [J. High Energy Phys. 03 (2014) 002] using a nonperturbative approach in the framework of QCD, and it reads $\sigma_{\text {tot }} \sim B \ln ^{2} s+C \ln s \ln \ln s$. We critically investigate if $B$ and $C$ can be obtained by means of best-fits to data for proton-proton and antiproton-proton scattering, including recent data obtained at the LHC, and also to data for other meson-baryon and baryon-baryon scattering processes. In particular, following the above-mentioned nonperturbative QCD approach, we also consider fits where the parameters $B$ and $C$ are set to $B=\kappa B_{\text {th }}$ and $C=\kappa C_{\text {th }}$, where $B_{\text {th }}$ and $C_{\text {th }}$ are universal quantities related to the QCD stable spectrum, while $\kappa$ (treated as an extra free parameter) is related to the asymptotic value of the ratio $\sigma_{\mathrm{el}} / \sigma_{\mathrm{tot}}$. Different possible scenarios are then considered and compared.
\end{abstract}

DOI: $10.1103 /$ PhysRevD.96.034015

\section{INTRODUCTION}

It is widely believed that the correct description of the strong interaction between quarks and gluons is provided by quantum chromodynamics (QCD), a belief supported by the numerous experimental verifications obtained in more than forty years. There are, however, energy regimes where the comparison between theory and experiment has not been performed yet, due to the lack of a theoretical prediction from the first principles of QCD. Such regimes are those where a major role is played by the nonperturbative dynamical aspects of QCD, which are notoriously very difficult to study. A successful comparison of experiment and theory in one of these regimes would certainly provide even stronger support to QCD being the appropriate description of strong interactions.

The total cross section $\left(\sigma_{\mathrm{tot}}\right)$ for hadron-hadron scattering processes at high energy is one of the best known observables for which a fully satisfactory prediction from first principles is not yet available. The reasons why the theoretical study of this quantity in QCD is extremely difficult is better understood recalling the relation between $\sigma_{\text {tot }}$ and the forward scattering amplitude provided by the optical theorem, which at high energy reads

\footnotetext{
*giordano@bodri.elte.hu enrico.meggiolaro@unipi.it ”precchia@ifi.unicamp.br
}

$$
\sigma_{\text {tot }}(s) \underset{s \rightarrow \infty}{=} \frac{\operatorname{Im} F(s, t=0)}{s},
$$

where $s$ is the total center-of-mass energy squared and $t=$ $-\vec{q}^{2}$ is the transferred momentum squared. The optical theorem shows that the study of $\sigma_{\text {tot }}$ requires the understanding of QCD in the regime of very low momentum transfer where the strong coupling constant becomes large [1], i.e., deep in the nonperturbative regime. On the other hand, at large $s$ one cannot employ the main tool used in nonperturbative investigations of QCD, namely the lattice formulation of the theory, to compute directly the relevant quantities. The most interesting features of $\sigma_{\text {tot }}$, which should be explained by QCD, are its increase with energy at large energy, and the fact that the leading term is apparently universal, i.e., independent of the type of particles initiating the process.

More generally, the same limitations discussed above apply to the study of soft high-energy hadron-hadron scattering, i.e., scattering at large $s$ and small $|t| \lesssim$ $1 \mathrm{GeV}^{2}$. Consequently, a full, model-independent description, obtained from the first principles of QCD, is not yet available for processes such as forward and near-forward elastic scattering. The study of soft high-energy physics has so far mostly proceeded through phenomenological models (for a recent review see Ref. [2]), on one side, and through general results obtained using basic properties of the theory, like unitarity and analyticity. Such general results 
are usually incorporated in the phenomenological models, for example exploiting them to set constraints on the functional form of observables concerning their dependence on energy and momentum transfer. Regarding hadronic total cross sections, the most important such result is probably the Froissart-Łukaszuk-Martin (FLM) bound [3-6], which states that $\sigma_{\text {tot }}$ cannot grow asymptotically faster than $\ln ^{2} s$, i.e.,

$$
\sigma_{\text {tot }}(s) \leq B_{\mathrm{FLM}} \ln ^{2}\left(s / s_{0}\right) \quad(s \rightarrow \infty),
$$

where $\sqrt{s_{0}}$ is an undetermined energy scale, and the coefficient $B_{\mathrm{FLM}}$ is also bounded,

$$
B_{\mathrm{FLM}} \leq \frac{\pi}{m_{\pi}^{2}} \approx 60 \mathrm{mb},
$$

with $m_{\pi}$ the $\pi^{0}$ mass. The FLM bound and the theory of Regge poles (see, e.g., Refs. [7,8]) are the inspiring principles behind the currently most successful phenomenological description of the experimental results for hadronic total cross sections (see below).

The recent measurements of $\sigma_{\text {tot }}$ at the Large Hadron Collider (LHC) at CERN have brought back the attention to the energy dependence of this quantity at high energy, with several new phenomenological analyses of the data (see Ref. [2] and references therein). Such measurements, made by the TOTEM Collaboration at $\sqrt{s}=7 \mathrm{TeV}$ and $\sqrt{s}=$ $8 \mathrm{TeV}$ [9-13] and by the ATLAS Collaboration at $\sqrt{s}=$ $7 \mathrm{TeV}$ [14], are currently the highest-energy measurements performed at colliders. Recently there have also been some advancements on the theoretical side. Two of us have obtained the leading behavior of the total cross section for meson-meson scattering [15] in the framework of the nonperturbative approach to soft high-energy scattering in QCD proposed long ago by Nachtmann [16], and later developed by several authors [17-21]. In this approach the relevant scattering amplitudes are related to the correlation functions (in the sense of the functional integral) of certain Wilson loops, describing the classical trajectories of the colliding mesons. It has been argued that similar correlation functions should be relevant to the description of scattering processes involving baryons as well [18], so the results of Ref. [15] should apply to general hadronic processes. Under certain assumptions, the leading asymptotic energy dependence turns out to be of the "Froissart" type, i.e., proportional to $\ln ^{2} s$, with a universal prefactor, independent of the type of particles involved. More precisely, the first two leading terms in energy read

$$
\sigma_{\text {tot }}^{a b}(s) \underset{s \rightarrow \infty}{\sim} B \ln ^{2}\left(\frac{s}{s_{0}^{a b}}\right)+C \ln \left(\frac{s}{s_{0}^{a b}}\right) \ln \left[\ln \left(\frac{s}{s_{0}^{a b}}\right)\right],
$$

where $\sigma_{\mathrm{tot}}^{a b}$ denotes the total cross section for the scattering of hadrons $a$ and $b$ with masses $m_{a}$ and $m_{b}$, respectively, and $s_{0}^{a b}=m_{a} m_{b}$. The coefficients $B$ and $C$ are equal to $\kappa B_{\text {th }}$ and $\kappa C_{\mathrm{th}}$, respectively, where $B_{\mathrm{th}}$ and $C_{\mathrm{th}}$ are universal quantities related to the QCD stable spectrum, while the coefficient $\kappa$ is, at the present stage, an undetermined real number with $0 \leq \kappa \leq 2$, connected to the asymptotic ratio between the total elastic cross section $\left(\sigma_{\mathrm{el}}\right)$ and the total cross section as $\sigma_{\mathrm{el}} / \sigma_{\mathrm{tot}}=\kappa / 2$. As experiments suggest universality of the $\ln ^{2} s$ term, it is natural to assume that $\kappa$ is universal as well. Making more detailed assumptions on $\kappa$, e.g., that a black-disk behavior is attained at high energy $(\kappa=1)$, it is then possible to provide a prediction for the coefficient of the leading term in $\sigma_{\text {tot }}$, which turns out to be in fair agreement with the experimental results. Since a fair amount of assumptions is involved in the derivation, this result cannot be claimed to be "the" prediction of QCD. Nevertheless, to the best of our knowledge, this is the closest to a prediction that one has come so far.

We point out that a subleading term of the form $\ln s$. $\ln \ln s$ has appeared in other approaches to the study of $\sigma_{\text {tot }}$, namely (i) in a bound for the "energy-averaged" total cross section obtained by Martin and Roy [22], (ii) in a revision of Heisenberg's model made by Nastase and Sonnenschein [23], where they assume that the Froissart bound is saturated, and (iii) in an improvement of the FLM bound in the AdS/CFT approach made by Díez et al. [24].

Regardless of its origin, the expression Eq. (4) provides a perfectly good parametrization of the total cross section at high energy, that can be used to fit the experimental results, treating $B$ and $C$ as fitting parameters. It is then legitimate to investigate whether in this way one can improve over the currently most successful parametrizations, where this term is absent. In particular, given that new experimental information is now available at higher energies, one may ask whether the asymptotic subleading contribution in Eq. (4) is already visible at LHC energies and, if so, how much this contribution is. The main purpose of the present analysis is precisely to test the functional form Eq. (4), by means of fits to $p p$ and $\bar{p} p$ data, and by comprehensive fits to all the available data for mesonbaryon and baryon-baryon scattering.

Besides this general purpose, our analysis allows us to test the viability of the various approaches leading to an expression like Eq. (4) for the total cross section. In particular, as already mentioned above, in the approach of Ref. [15] $B$ and $C$ are related to the QCD spectrum and to the elastic-to-total cross section ratio: this puts severe constraints on the allowed values of $B$ and $C$. In pursuing this line of investigation, the coefficients $B$ and $C$ can either be fixed to their theoretical prediction, or treated as free parameters when fitting the experimental data.

The paper is organized as follows. In Sec. II we present a summary of some useful basic relations, and the results of Ref. [15] that constitute the basis of the present analysis. In Sec. III we discuss the parametrization used in the fits and in Sec. IV we detail the data set considered. In Sec. V we 
present and discuss the results obtained in the fits. Finally, we present our conclusions in Sec. VI.

\section{SUMMARY OF THEORETICAL RESULTS}

In this section we present a brief summary of the theoretical results of Ref. [15] concerning the asymptotic high-energy behavior of the elastic hadron-hadron scattering amplitude.

The approach employed in Ref. [15] is that originally proposed by Nachtmann [16] for elastic quark-quark scattering, and later expanded by several authors to describe hadron-hadron scattering [17-21]. In this approach, the elastic scattering amplitude in the soft high-energy regime $\left(s \rightarrow \infty,|t| \lesssim 1 \mathrm{GeV}^{2}\right)$ of two mesons $a$ and $b$ with masses $m_{a}$ and $m_{b}$, respectively, is obtained from the elastic scattering amplitude of two colorless dipoles of fixed transverse size, after folding with appropriate wave functions describing the colliding mesons. In turn, the dipole-dipole scattering amplitude is obtained from the (normalized, connected) correlation function of two Wilson loops in Minkowski space, running along the classical trajectories of the dipoles, in the limit of infinite longitudinal extension of the loops. Exploiting analytic continuation [25-31], it is possible to reconstruct these correlation functions from their Euclidean counterparts, which in turn can be related to the QCD spectrum by appropriately inserting a complete set of states between the two Wilson-loop operators. Under certain analyticity assumptions, discussed in detail in Ref. [15], it is then possible to derive the asymptotic behavior of the Minkowskian correlators at large energy and large impact parameter (which is the transverse distance between the classical trajectories of the incident particles), from which the elastic amplitude and the total cross section are finally obtained. The same chain of arguments can be used in the case of processes involving baryons, since similar Wilsonloop correlation functions are involved in the calculation of the corresponding scattering amplitudes [18], and the detailed transverse geometry of the Wilson loops plays no role in the derivation of Ref. [15]. The results discussed below are therefore expected to be valid in more general hadronic processes than just meson-meson scattering. For more details, we invite the interested reader to confer the original references.

In Ref. [15] the elastic scattering amplitude was computed assuming that for asymptotically large energies one finds a black-disk behavior, corresponding to the Minkowskian Wilson-loop correlator tending to zero as the energy goes to infinity, for sufficiently large but fixed impact parameter. It is straightforward to generalize this result to the case where this correlator tends to a nonzero constant $\kappa-1$, which we assume to be independent of the transverse size of the loops. Analiticity requires $\kappa$ to be real, and unitarity then requires that $\kappa \in[0,2]$. One then finds

$$
F^{a b}(s, t) \underset{s \rightarrow \infty, t \rightarrow 0}{\sim} 4 \pi i s \kappa\left(\frac{\eta}{\tilde{m}}\right)^{2} \frac{J_{1}(x)}{x},
$$

if $x \equiv \eta \sqrt{-t} / \tilde{m}$ is kept fixed. Here

$$
\begin{aligned}
\eta= & \frac{1}{2} W\left(2 e^{2(\tilde{s}-1) \chi}\right)=(\tilde{s}-1) \chi-\frac{1}{2} \ln [(\tilde{s}-1) \chi] \\
& +\frac{\ln [(\tilde{s}-1) \chi]}{4(\tilde{s}-1) \chi}+\ldots,
\end{aligned}
$$

with $W$ the Lambert $W$ function [32], and $\chi=\ln \left(s / s_{0}^{a b}\right)$ with $s_{0}^{a b}=m_{a} m_{b}$. In the equations above, $\tilde{s}$ and $\tilde{m}$ are, respectively, the spin and mass of the particle in the QCD stable spectrum that maximizes the ratio

$$
l_{p} \equiv \frac{s_{p}-1}{m_{p}} \quad\left(s_{p}>1\right),
$$

where $s_{p}$ and $m_{p}$ are the spin and the mass of particle $p$. By "QCD stable spectrum" we mean here all those particles that are stable when strong interactions are considered in isolation. The assumed independence of $\kappa$ on the transverse size of the dipoles implies that the scattering amplitude Eq. (5), obtained after trivially folding with the hadronic wave functions, is a universal function of $x$.

Taking the limit $t \rightarrow 0$ in Eq. (5) and using the optical theorem Eq. (1), we get for the total cross section

$$
\sigma_{\mathrm{tot}}^{a b}(s) \rightarrow \frac{2 \pi}{\tilde{m}^{2}} \kappa \eta^{2}+\mathcal{O}(\eta) .
$$

Using now Eq. (6), we obtain, up to first subleading order,

$$
\begin{aligned}
\sigma_{\text {tot }}^{a b}(s) \rightarrow & B \ln ^{2}\left(\frac{s}{s_{0}^{a b}}\right)+C \ln \left(\frac{s}{s_{0}^{a b}}\right) \ln \left[\ln \left(\frac{s}{s_{0}^{a b}}\right)\right] \\
& +\mathcal{O}\left(\ln \left(s / s_{0}^{a b}\right)\right),
\end{aligned}
$$

with

$$
\begin{aligned}
& B=\kappa B_{\mathrm{th}}, \quad B_{\mathrm{th}}=2 \pi \frac{(\tilde{s}-1)^{2}}{\tilde{m}^{2}}, \\
& C=\kappa C_{\mathrm{th}}, \quad C_{\mathrm{th}}=-2 \pi \frac{(\tilde{s}-1)}{\tilde{m}^{2}} .
\end{aligned}
$$

Notice the relations

$$
\frac{B}{C}=1-\tilde{s}, \quad 2 \pi \frac{B}{C^{2}}=\frac{\tilde{m}^{2}}{\kappa} .
$$

[For comparison, we recall here two other theoretical predictions for the ratio $B / C$, obtained using completely different approaches: in Ref. [23] the value $B / C=-1 / 2$ is found, which agrees with the first Eq. (11) when $\tilde{s}=3 / 2$, while in Ref. [24] the value $B / C=-1 / 4$ is derived.] 
We then find that in the limit $s \rightarrow \infty$ both the leading and the subleading term in the total cross section are independent of the scattering particles.

We also want to recall that in Ref. [15] another subleading term of order $\mathcal{O}(\ln s)$, i.e., $Q^{a b} \ln \left(s / s_{0}^{a b}\right)$, was also found (and such a term is also present in the analysis of Ref. [24]). Contrary to the leading and subleading terms discussed above, the coefficient $Q^{a b}$ is expected to depend on the colliding particles, even if our assumption on $\kappa$ is met. ${ }^{1}$

In Ref. [15] the values of $B_{\text {th }}$ and $C_{\text {th }}$ were estimated by maximizing the ratio Eq. (7) over the higher-spin, QCD stable spectrum. The resulting "dominant" particle was found to be the $\Omega^{ \pm}$baryon, with mass $m_{\Omega^{ \pm}} \approx 1.67 \mathrm{GeV}$ and spin 3/2, that yields [using Eq. (10)]

$$
B_{\mathrm{th}}^{\Omega}=0.22 \mathrm{mb}, \quad C_{\mathrm{th}}^{\Omega}=-2 B_{\mathrm{th}}^{\Omega}=-0.44 \mathrm{mb} .
$$

One of the assumptions in Nachtmann's approach is that the processes of splitting and annihilation of partons inside hadrons can be neglected over a small time window around collision time [16]. The description of hadrons in terms of dipoles is therefore perhaps better justified in the quenched limit of the theory. In this case the relevant spectrum over which one has to maximize Eq. (7) is the glueball spectrum of the pure-gauge theory. The states of interest here (for details see Ref. [15]) are the $J^{P C}=2^{++}$glueball state, with mass $m_{g\left(2^{++}\right)} \approx 2.40 \mathrm{GeV}$, and the $3^{+-}$, with mass $m_{g\left(3^{+-}\right)} \approx 3.55 \mathrm{GeV}$, both calculated in the quenched approximation [33], for which one finds

$B_{\mathrm{th}}^{g\left(2^{++}\right)}=0.42 \mathrm{mb}, \quad C_{\mathrm{th}}^{g\left(2^{++}\right)}=-B_{\mathrm{th}}^{g\left(2^{++}\right)}=-0.42 \mathrm{mb}$,

and

$B_{\mathrm{th}}^{g\left(3^{+-}\right)}=0.78 \mathrm{mb}, \quad C_{\mathrm{th}}^{g\left(3^{+-}\right)}=-\frac{1}{2} B_{\mathrm{th}}^{g\left(3^{+-}\right)}=-0.39 \mathrm{mb}$.

The value of $B_{\text {th }}$ obtained using the $\Omega^{ \pm}$baryon is the closest to the values of $B$ published in the Review of Particle Physics by the Particle Data Group (PDG). In the 2014 edition, fits to forward quantities using energies $\sqrt{s} \geq$ $5 \mathrm{GeV}$ yielded $B_{\mathrm{PDG}}=0.2704 \pm 0.0038 \mathrm{mb}$ [34]; in the 2016 edition the revised value is $0.2720 \pm 0.0024 \mathrm{mb}$,

\footnotetext{
${ }^{1}$ It is worth mentioning that $Q^{a b}$ is sensitive to the choice of scale in the $\ln ^{2} s$ term. For example, if a fixed, reactionindependent scale $s_{0}$ were used in Eq. (9) instead of $s_{0}^{a b}$, then $Q^{a b}$ would trivially depend on the reaction. What is meant here is that $Q^{a b}$ is expected to be reaction-dependent even with our particular (reaction-dependent) choice of scale in the $\ln ^{2} s$ term.
}

obtained from a fit to data with $\sqrt{s} \geq 5 \mathrm{GeV}$ and including the most recent measurements at the LHC [35].

From the amplitude Eq. (5) one can easily calculate the asymptotic behavior of the total elastic cross section $\sigma_{\mathrm{el}}^{a b}$ using

$$
\begin{aligned}
\sigma_{\mathrm{el}}^{a b}(s) & =\int_{-\infty}^{0} d t \frac{d \sigma_{\mathrm{el}}^{a b}}{d t}(s, t), \\
\frac{d \sigma_{\mathrm{el}}^{a b}}{d t}(s, t) & =\frac{1}{16 \pi s^{2}}\left|F^{a b}(s, t)\right|^{2},
\end{aligned}
$$

and assuming that the small- $t$ region gives the dominant contribution. From this result and from Eq. (9), we obtain

$$
\frac{\sigma_{\mathrm{el}}^{a b}}{\sigma_{\mathrm{tot}}^{a b}} \sim \frac{\kappa}{2}(s \rightarrow \infty) .
$$

Equations (5) and (16) show that, if $\kappa<1$ (i.e., $\left.\sigma_{\mathrm{el}} / \sigma_{\mathrm{tot}}<1 / 2\right)$, the elastic scattering amplitude $F^{a b}(s, t)$ behaves asymptotically as a grey disk with constant profile function equal to $\kappa$ and radius growing as $\ln s{ }^{2}$ If $\kappa=1$ we have the so-called black disk $\left(\sigma_{\mathrm{el}} / \sigma_{\mathrm{tot}}=1 / 2\right)$, and if $\kappa>1$ we have the antishadowing regime $\left(\sigma_{\text {el }} / \sigma_{\text {tot }}>1 / 2\right)[37,38]$.

We want to stress the fact that so far we have assumed that $\kappa$ is a universal parameter. Although this assumption is made plausible by the observed universality of total cross sections at high energy, a more general analysis should start with a particle-dependent parameter, $\kappa^{a b}$, and universality should then emerge from the fit results rather than being imposed a priori. This would however increase considerably the number of fitting parameters. Therefore, even if this case is not the most general one, we will consider $\kappa$ as a universal parameter in the present analysis.

\section{PARAMETRIZATION OF THE TOTAL CROSS SECTION}

In this work we want to study the leading and subleading high-energy behavior of hadron-hadron total cross sections using a best-fit analysis of hadronic scattering data. The available data of highest energy (excluding cosmic rays) are those for $p p$ scattering at $8 \mathrm{TeV}$ at the $\mathrm{LHC}[13,39,40]$ and for $\bar{p} p$ scattering at $1.8 \mathrm{TeV}$ at the Tevatron [41-43]. If we want to include also data from other meson-baryon and baryon-baryon scattering processes (which are available

\footnotetext{
${ }^{2} \mathrm{We}$ remind the reader that if one expresses the elastic amplitude $F(s, t)$ in terms of the profile function $\Gamma(s, b)$, i.e. (assuming azimuthal symmetry) [8,36], $F(s, t)=$ $4 \pi i s \int_{0}^{\infty} d b b J_{0}(b \sqrt{-t}) \Gamma(s, b)$, with $b$ the absolute value of the impact parameter, then the so-called grey disk of radius $R(s)$ is a simple model in which $\Gamma_{\mathrm{GD}}(s, b)=\Gamma_{0}(s)$ for $0 \leq b \leq R(s)$, and $\Gamma_{\mathrm{GD}}(s, b)=0$ for $b>R(s)$, which leads to $F_{\mathrm{GD}}(s, t)=$ $4 \pi i s R(s) \Gamma_{0}(s) J_{1}(R(s) \sqrt{-t}) / \sqrt{-t}$ and, using Eq. (15) and the optical theorem Eq. (1), to $\sigma_{\mathrm{el}} / \sigma_{\mathrm{tot}}=\Gamma_{0}(s) / 2$.
} 
only for $\sqrt{s} \lesssim 25 \mathrm{GeV}$ ), we need a parametrization for the low-energy region as well. In this section we present our parametrization for the total cross section, and we fix the notation for the best-fit variants considered in our analysis.

We will divide the total cross section in two parts, aimed at describing the behavior in the "low-energy" (LE) and the "high-energy" (HE) regions, respectively,

$$
\sigma_{\mathrm{tot}}(s)=\sigma_{\mathrm{LE}}(s)+\sigma_{\mathrm{HE}}(s) .
$$

As usual in the literature, we parametrize the energy dependence of $\sigma_{\mathrm{tot}}(s)$ at low energy by means of Reggeon exchange in the $t$-channel $[7,8]$. The Reggeonexchange contribution reads

$$
R_{i}^{a b}(s)=A_{i}^{a b}\left(\frac{s}{s_{0}^{a b}}\right)^{-b_{i}} \quad(i=1,2),
$$

where $A_{i}^{a b}$ is associated to the residue function and $b_{i}$ to the intercept of the Reggeon trajectory $\alpha_{i}(t)$, i.e., $b_{i}=1-\alpha_{i}(0)$. We consider two Reggeon contributions: the index $i=1$ corresponds to a trajectory with even signature, while $i=2$ corresponds to a trajectory with odd signature. The latter contributes with a minus sign to $a b$ scattering and with a plus sign to the crossed channel, $\bar{a} b$. Summarizing, the low-energy parametrization reads ${ }^{3}$

$$
\sigma_{\mathrm{LE}}^{a^{ \pm} b}(s)=A_{1}^{a b}\left(\frac{s}{s_{0}^{a b}}\right)^{-b_{1}} \mp A_{2}^{a b}\left(\frac{s}{s_{0}^{a b}}\right)^{-b_{2}}+A_{\mathbb{P}}^{a b},
$$

where we have also added a constant term $A_{\mathbb{P}}^{a b}$ [which is usually understood as the contribution of the critical Pomeron, i.e., a simple pole in the complex angularmomentum plane with intercept $\left.\alpha_{\mathbb{P}}(0)=1\right] .{ }^{4}$ The highenergy contribution is parametrized by adding to the leading $\left(\sim \ln ^{2} s\right)$ and subleading $(\sim \ln s \cdot \ln \ln s)$ terms also another subleading term of the form $\sim \ln s$, i.e.,

$$
\begin{aligned}
\sigma_{\mathrm{HE}}^{a^{ \pm} b}(s)= & \kappa\left\{B \ln ^{2}\left(\frac{s}{s_{0}^{a b}}\right)+C \ln \left(\frac{s}{s_{0}^{a b}}\right) \ln \left[\ln \left(\frac{s}{s_{0}^{a b}}\right)\right]\right\} \\
& +Q^{a b} \ln \left(\frac{s}{s_{0}^{a b}}\right),
\end{aligned}
$$

where, following the analysis of Ref. [15] (briefly recalled in Sec. II), $B$ and $C$ are treated as universal parameters,

\footnotetext{
${ }^{3}$ In the left-hand side of the equation, $a^{+} \equiv a$ represents a positive-charge particle, while $a^{-} \equiv \bar{a}$ corresponds to its negative-charge antiparticle.

${ }^{4}$ Actually, a constant term $A_{\mathbb{P}}^{a b}$ could also originate from the sum of the critical (i.e., simple-pole) Pomeron with the constant term generated by higher-order singularities, e.g., a triple-pole Pomeron (which also gives a $\ln ^{2} s$ term). In any case, however, its value is obviously affected by the choice of the energy scale in the leading and subleading terms in Eq. (20) (see also the discussion at the end of this section).
}

while $Q^{a b}$, as well as $A_{1}^{a b}, A_{2}^{a b}$ and $A_{\mathbb{P}}^{a b}$, are reactiondependent. As already said at the end of Sec. II, we shall also assume (as in Ref. [15]) that $\kappa$ is independent of the properties of the scattering particles. We consider the energy scale to be a fixed parameter and to depend only on the masses of the scattering particles, $s_{0}^{a b}=m_{a} m_{b}$. Moreover, $Q^{a b}$ are taken to be crossing-symmetric, i.e., $Q^{\bar{a} b}=Q^{a b}$.

Summarizing, $A_{i}^{a b}(\mathrm{mb}), b_{i}$ (dimensionless) and $A_{\mathbb{P}}^{a b}$ $(\mathrm{mb})$ are always free parameters to be determined in the fits. The parameters $B(\mathrm{mb}), C(\mathrm{mb}), Q^{a b}(\mathrm{mb})$ and $\kappa$ (dimensionless) can be fixed or free, as detailed below in the descriptions of our variants of fits.

The names of the variants are written using the following notation: LT stands for leading term, SLT for sub leading term, the subscript "th" refers to the case where we fix $B$ $\left(\mathrm{LT}_{\mathrm{th}}\right)$ or both $B$ and $C$ ( $\left.\mathrm{SLT}_{\mathrm{th}}\right)$ to the theoretical values discussed in Sec. II, and the subscript " $\kappa$ " indicates that the parameter $\kappa$ is free. The main variants considered here are described below. By default, the coefficient $Q^{a b}$ of the logarithmic term is fixed to zero: in those cases in which a nonzero logarithmic term is considered ( $Q^{a b}$ free), we shall add a "Q" in front of the variant name.

(i) LT: $\kappa=1, C=0$ and $Q=0$ are fixed parameters, while $B$ is free. This case (in which the subleading terms are absent) corresponds essentially to the parametrization used by the PDG in their analysis of forward data [35] and to the highest-rank result obtained by the COMPETE Collaboration [44] (see below for more details). As mentioned above, when $Q$ is included as a free parameter, we shall denote this variant as QLT. The same rule also applies to the other variants.

(ii) SLT: $\kappa=1$ and $Q=0$ are fixed parameters, while $B$ and $C$ are free parameters. This case corresponds to the previous parametrization with the inclusion of the subleading term.

(iii) $\mathrm{LT}_{\text {th }}: \kappa=1, B=B_{\text {th }}, C=0$ and $Q=0$ are fixed parameters. This variant has $B$ fixed to the theoretical values discussed in Sec. II and no subleading term.

(iv) $\mathrm{SLT}_{\mathrm{th}}: \kappa=1, B=B_{\mathrm{th}}, C=C_{\mathrm{th}}$ and $Q=0$ are fixed parameters. This variant has both $B$ and $C$ fixed to the theoretical values discussed in Sec. II.

(v) $\mathrm{SLT}_{\text {th }, \kappa}: B=B_{\text {th }}, C=C_{\text {th }}$ and $Q=0$ are fixed parameters, while $\kappa$ is a free parameter.

\footnotetext{
${ }^{5}$ This means that we are neglecting here a possible "odderon" contribution to the total cross sections. Notice that also the PDG and the highest-rank COMPETE parametrizations of the total cross sections are crossing-symmetric. Moreover, we recall that, with the usual choice of rotation-invariant dipole wave functions [17], the high-energy scattering amplitudes, derived using the theoretical approach briefly summarized in Sec. II, turn out to be crossing-symmetric [30].
} 
The main difference between our LT parametrization, the highest-rank result by COMPETE, and the PDG parametrization, is in the energy scale appearing in the leading term $\ln ^{2} s$. In the COMPETE analysis, the energy scale $s_{0}$ is a free parameter, which does not depend on the scattering particles. Our energy scale, on the other hand, is fixed and depends only on the masses of the scattering particles, $s_{0}^{a b}=m_{a} m_{b}$. In the PDG analysis this scale depends on the masses of the colliding particles and on a universal mass scale also entering their parametrization of the coefficient $B$, so it contains both a fixed and a free part. All in all, this results in the three parametrizations differing pairwise by a particle-dependent term of order $\mathcal{O}(\ln s)$ (besides a particledependent constant term that can be reabsorbed in $A_{\mathbb{P}}^{a b}$ ). In other words, and more precisely, if we denote by $\tilde{s}_{0}^{a b}$ the scale used in the PDG or in the COMPETE parametrizations, these correspond to our QLT parametrization with $Q^{a b}$ fixed to $Q^{a b}=2 B \ln \frac{s_{0}^{a b}}{\tilde{s}_{0}^{a b}}$ (up to a redefinition of $A_{\mathbb{P}}^{a b}$ ). Since very high-energy data are not yet available, this can affect the result obtained for $B$ in the fits.

\section{DATA SET AND METHODOLOGY}

In this section we describe our data set and the methodology used in our fits.

\section{A. Data set}

Our data set comprises data from meson-baryon and baryon-baryon scattering, namely $p p, \bar{p} p, p n, \bar{p} n, \pi^{ \pm} p$, $K^{ \pm} p, K^{ \pm} n$, with a cutoff energy $\sqrt{s_{\min }}=5 \mathrm{GeV}$ (so that we are well above the resonance region). This cutoff energy is the same used in the highest-rank result by the COMPETE Collaboration [44] and in the PDG analysis [35]. Only data obtained in accelerator experiments were included in the fits, i.e., no cosmic rays data were considered, and the data sets are those available at the PDG website [45].

For $p p$ scattering, besides the data already in the PDG data set at $7 \mathrm{TeV}$, we have included further data at 7 and $8 \mathrm{TeV}$ obtained by the TOTEM and ATLAS Collaborations, namely, the luminosity-independent measurement at $7 \mathrm{TeV}$ [11], the first measurement at $8 \mathrm{TeV}$ [12] and the values of $\sigma_{\text {tot }}$ obtained together with the $\rho$ value at $8 \mathrm{TeV}$ from the Coulomb-Nuclear interference region in the differential cross section data [13] by the TOTEM Collaboration, and the measurement at $7 \mathrm{TeV}$ by the ATLAS Collaboration [14]. This information is summarized in Table I. Our data set therefore comprises data with energy in the range $5 \mathrm{GeV} \leq \sqrt{s} \leq 8 \mathrm{TeV}$.

Cosmic-ray data [46-48] are shown in the figures just to illustrate the trend with energy and, as stated above, they have not been included in the fits. Since their uncertainties are large, we expect that their inclusion in the fits would not change much the results. The energy range and the number
TABLE I. Experimental data of $\sigma_{\text {tot }}$ in the LHC energy region.

\begin{tabular}{lcc}
\hline \hline$\sqrt{s}(\mathrm{TeV})$ & $\sigma_{\text {tot }}(\mathrm{mb})$ & Collaboration \\
\hline 7 & $98.3 \pm 2.8$ & TOTEM [9] \\
7 & $98.6 \pm 2.2$ & TOTEM [10] \\
7 & $98.0 \pm 2.5$ & TOTEM [11] \\
7 & $99.1 \pm 4.1$ & TOTEM [11] \\
7 & $95.4 \pm 1.4$ & ATLAS [14] \\
8 & $101.7 \pm 2.9$ & TOTEM [12] \\
8 & $102.9 \pm 2.3$ & TOTEM [13] \\
8 & $103.0 \pm 2.3$ & TOTEM [13] \\
\hline \hline
\end{tabular}

of points available for each scattering channel that we have considered are shown in Table II.

In all the cases, we have treated the data points as independent, including those that have the same energy. For all data we have considered statistic and systematic uncertainties added in quadrature.

We stress that we are not including data from reactions that involve photons or deuterons, and we do not constrain our fits using the data for the $\rho$ parameter, as it is done by COMPETE and PDG.

Finally, we mention that there are 9 points available for $\Sigma^{-} p$ scattering in the energy region of interest [45]. Including these points makes the fits more unstable (due to the absence of data in the corresponding crossed channel), but this does not affect the results, because of their large errors. We have therefore decided not to include them in our analysis.

\section{B. Methodology}

We first consider fits using our parametrization, Eqs. (17) and (19)-(20), to data from $p p$ and $\bar{p} p$ scattering only. This pair of reactions constitutes the set with available data in the largest energy range. Since the subleading term of interest here is expected to be relevant at high energies, it is important to estimate its contribution without much weight

TABLE II. Information about the reactions in our data set: minimum energy, maximum energy and number of points for each reaction.

\begin{tabular}{lccc}
\hline \hline Reaction & $\sqrt{s_{\min }}(\mathrm{GeV})$ & $\sqrt{s_{\max }}(\mathrm{GeV})$ & Number of points \\
\hline$p p$ & 5.01 & 8000 & 112 \\
$\bar{p} p$ & 5.16 & 1800 & 59 \\
$p n$ & 5.30 & 26.40 & 34 \\
$\bar{p} n$ & 5.18 & 22.98 & 33 \\
$\pi^{+} p$ & 5.21 & 25.28 & 50 \\
$\pi^{-} p$ & 5.03 & 34.67 & 95 \\
$K^{+} p$ & 5.13 & 24.14 & 40 \\
$K^{-} p$ & 5.11 & 24.14 & 63 \\
$K^{+} n$ & 5.24 & 24.16 & 28 \\
$K^{-} n$ & 5.11 & 24.16 & 36 \\
& & Total: & 559 \\
\hline \hline
\end{tabular}




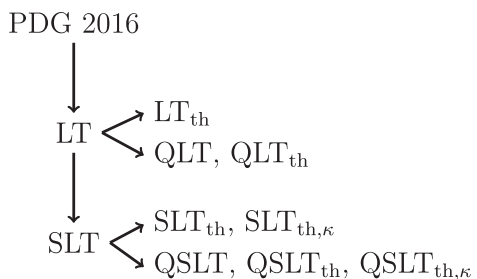

FIG. 1. Initial-value scheme used in the fits.

from the low-energy data from other reactions. Nevertheless, we have also considered fits to all the data for the hadron-hadron processes shown in Table II.

In order to start from a solid and updated result, we decided to use as initial values for the LT fit the results presented in PDG 2016 [35], and then use the results of LT as initial values for the SLT fit. In this way, fitting first LT (that essentially corresponds to the PDG parametrization,

TABLE III. Results of fits with LT, SLT and QLT to $\sigma_{\text {tot }}$ data of $p p$ and $\bar{p} p$ scattering. Parameters $A_{1}, A_{2}, A_{\mathbb{P}}, B, C$ and $Q$ are in $\mathrm{mb}$, while $b_{1}, b_{2}$ and $\kappa$ are dimensionless.

\begin{tabular}{lccc}
\hline \hline & \multicolumn{3}{c}{ Fits to $\sigma_{\text {tot }}$} \\
\cline { 2 - 4 } & LT & SLT & QLT \\
\hline$B$ & $0.2269(38)$ & $0.349(29)$ & $0.311(19)$ \\
$C$ & 0 (fixed) & $-0.95(21)$ & 0 (fixed) \\
$\kappa$ & 1 (fixed) & 1 (fixed) & 1 (fixed) \\
$Q$ & 0 (fixed) & 0 (fixed) & $-2.40(48)$ \\
$b_{1}$ & $0.342(15)$ & $0.560(76)$ & $0.586(89)$ \\
$b_{2}$ & $0.539(15)$ & $0.541(16)$ & $0.541(16)$ \\
$A_{1}$ & $56.8(1.7)$ & $64.4(8.2)$ & $60.6(8.7)$ \\
$A_{2}$ & $35.2(2.5)$ & $35.6(2.5)$ & $35.6(2.5)$ \\
$A_{\mathbb{P}}$ & $24.77(60)$ & $35.7(2.0)$ & $41.7(3.0)$ \\
$\chi^{2} / \nu$ & 0.972 & 0.933 & 0.934 \\
$\nu$ & 165 & 164 & 164 \\
\hline \hline
\end{tabular}

as we have already observed above) we create a reference for discussing differences when we include the subleading term as well (SLT), instead of comparing directly to the PDG result. This procedure, however, turned out to be problematic in the fit to all hadronic data (see below Sec. V B 1). In that case we therefore decided to use as initial values for the parameters $B$ and $C$ in the SLT fit to all data the results obtained in the SLT fit to $p p / \bar{p} p$ data. For the other parameters, we used the results obtained in the LT fit to all data. The detailed scheme is shown in Fig. 1, where $X \rightarrow Y$ means that the results of variant $X$ were used as initial values for the fit with variant $Y$.

We use the reduced chi-squared $\left(\chi^{2} / \nu\right.$, where $\nu$ is the number of degrees of freedom) as a measure of the quality of the fit $[49,50]$. However, we do not base our preference for a result over another on small differences in the value of this quantity, since the inclusion of both statistical and systematic uncertainties puts some limits on the use of this test. It is important to note that some data obtained by the TOTEM Collaboration do not have statistical uncertainty (as can be seen in the caption of Table 1 in Ref. [11]).

The fits were performed with the class TMinuit from the ROOT framework [51] with $1 \sigma$ of confidence level.

\section{FIT RESULTS}

In this section we present the results obtained in our fits, first to $p p$ and $\bar{p} p$ data only, and later to all reactions, considering all variants described above in Sec. III. Finally, we compare and discuss our results.

\section{A. Fits to $p p$ and $\bar{p} p$ data}

The parameters obtained in fits to $p p$ and $\bar{p} p$ data with LT, SLT and QLT are shown in Table III, those with $\mathrm{LT}_{\text {th }}$, $\mathrm{SLT}_{\text {th }}$ and $\mathrm{SLT}_{\text {th }, \kappa}$ in Table IV and those with QLT $_{\text {th }}$, QSLT $_{\text {th }}$ and QSLT $_{\text {th }, \kappa}$ in Table V. The curves calculated

TABLE IV. Results of fits with $\mathrm{LT}_{\text {th }}\left(\kappa=1, B=B_{\text {th }}, C=0, Q=0\right), \operatorname{SLT}_{\text {th }}\left(\kappa=1, B=B_{\text {th }}, C=C_{\text {th }}, Q=0\right)$ and SLT th, $(\kappa$ free, $B=B_{\mathrm{th}}, C=C_{\mathrm{th}}, Q=0$ ) to $\sigma_{\mathrm{tot}}$ data of $p p$ and $\bar{p} p$ scattering. The values of $B$ and $C$ are fixed to the theoretical values calculated with the masses and the spins of the $\Omega^{ \pm}$baryon, the $2^{++}$glueball state and the $3^{+-}$glueball state (quenched values), while the parameter $Q$ is fixed to zero. For the units of measurement of the parameters, see Table III.

\begin{tabular}{|c|c|c|c|c|c|}
\hline & \multicolumn{3}{|c|}{$\Omega^{ \pm}$baryon } & \multirow{2}{*}{$\frac{2^{++} \text {glueball }}{\mathrm{SLT}_{\text {th }, \kappa}}$} & \multirow{2}{*}{$\frac{3^{+-} \text {glueball }}{\mathrm{SLT}_{\mathrm{th}, \kappa}}$} \\
\hline & $\mathrm{LT}_{\mathrm{th}}$ & $\mathrm{SLT}_{\text {th }}$ & $\mathrm{SLT}_{\mathrm{th}, \kappa}$ & & \\
\hline$B_{\text {th }}$ & 0.22 (fixed) & 0.22 (fixed) & 0.22 (fixed) & 0.42 (fixed) & 0.78 (fixed) \\
\hline$C_{\mathrm{th}}$ & 0 (fixed) & -0.44 (fixed) & -0.44 (fixed) & -0.42 (fixed) & -0.39 (fixed) \\
\hline$\kappa$ & 1 (fixed) & 1 (fixed) & $1.377(18)$ & $0.6159(96)$ & $0.3097(51)$ \\
\hline$Q$ & 0 (fixed) & 0 (fixed) & 0 (fixed) & 0 (fixed) & 0 (fixed) \\
\hline$b_{1}$ & $0.365(10)$ & $0.743(20)$ & $0.458(20)$ & $0.385(17)$ & $0.361(17)$ \\
\hline$b_{2}$ & $0.539(15)$ & $0.528(16)$ & $0.540(15)$ & $0.539(15)$ & $0.539(15)$ \\
\hline$A_{1}$ & $58.5(1.7)$ & $115.3(8.5)$ & $57.5(3.2)$ & $56.0(2.2)$ & $56.3(2.0)$ \\
\hline$A_{2}$ & $35.3(2.5)$ & $33.7(2.4)$ & $35.4(2.5)$ & $35.3(2.4)$ & $35.2(2.4)$ \\
\hline$A_{\mathbb{P}}$ & $25.75(21)$ & $35.862(74)$ & $32.17(29)$ & $28.13(46)$ & $26.38(55)$ \\
\hline$\chi^{2} / \nu$ & 0.987 & 3.59 & 0.937 & 0.957 & 0.965 \\
\hline$\nu$ & 166 & 166 & 165 & 165 & 165 \\
\hline
\end{tabular}


TABLE V. Results of fits with $\mathrm{QLT}_{\text {th }}\left(\kappa=1, B=B_{\text {th }}, C=0, Q\right.$ free $), \mathrm{QSLT}_{\text {th }}\left(\kappa=1, B=B_{\mathrm{th}}, C=C_{\text {th }}, Q\right.$ free $)$ and QSLT ${ }_{\text {th }, \kappa}(\kappa$ free, $B=B_{\text {th }}, C=C_{\text {th }}, Q$ free) to $\sigma_{\text {tot }}$ data of $p p$ and $\bar{p} p$ scattering. The values of $B$ and $C$ are fixed to the theoretical values calculated with the masses and the spins of the $\Omega^{ \pm}$baryon, the $2^{++}$glueball state and the $3^{+-}$glueball state (quenched values). For the units of measurement of the parameters, see Table III.

\begin{tabular}{|c|c|c|c|c|c|}
\hline & \multicolumn{3}{|c|}{$\Omega^{ \pm}$baryon } & \multirow{2}{*}{$\frac{2^{++} \text {glueball }}{\text { QSLT }_{\mathrm{th}, \kappa}}$} & \multirow{2}{*}{$\frac{3^{+-} \text {glueball }}{\text { QSLT }_{\text {th }, \kappa}}$} \\
\hline & $\mathrm{QLT}_{\text {th }}$ & $\mathrm{QSLT}_{\mathrm{th}}$ & $\mathrm{QSLT}_{\mathrm{th}, \kappa}$ & & \\
\hline$B_{\mathrm{th}}$ & 0.22 (fixed) & 0.22 (fixed) & 0.22 (fixed) & 0.42 (fixed) & 0.78 (fixed) \\
\hline$C_{\mathrm{th}}$ & 0 (fixed) & -0.44 (fixed) & -0.44 (fixed) & -0.42 (fixed) & -0.39 (fixed) \\
\hline$\kappa$ & 1 (fixed) & 1 (fixed) & $1.53(10)$ & $0.771(49)$ & $0.407(25)$ \\
\hline$Q$ & $0.19(12)$ & $1.86(13)$ & $-0.69(41)$ & $-1.58(45)$ & $-2.00(47)$ \\
\hline$\widetilde{b}_{1}$ & $0.335(21)$ & $0.311(18)$ & $0.566(82)$ & $0.576(86)$ & $0.581(88)$ \\
\hline$b_{2}$ & $0.539(15)$ & $0.538(14)$ & $0.541(16)$ & $0.541(16)$ & $0.541(16)$ \\
\hline$A_{1}$ & $57.9(1.5)$ & $63.3(1.3)$ & $63.2(8.0)$ & $61.8(8.3)$ & $61.2(8.5)$ \\
\hline$A_{2}$ & $35.2(2.5)$ & $35.1(2.3)$ & $35.6(2.5)$ & $35.6(2.5)$ & $35.6(2.5)$ \\
\hline$A_{\mathbb{P}}$ & $23.4(1.6)$ & $16.0(1.8)$ & $37.4(3.0)$ & $39.6(3.0)$ & $40.7(3.0)$ \\
\hline$\chi^{2} / \nu$ & 0.977 & 0.985 & 0.933 & 0.934 & 0.934 \\
\hline$\nu$ & 165 & 165 & 164 & 164 & 164 \\
\hline
\end{tabular}

with the parameters of LT, SLT and QLT are compared to the experimental data in Fig. 2, and those of $\mathrm{SLT}_{\mathrm{th}, \kappa}$ and QSLT $_{\text {th }, \kappa}$ in Figs. 3 and 4, respectively. Below we discuss the results that we have obtained using the different variants.

\section{Fits with $L T$ and SLT}

The results obtained with variants LT and SLT provide a good description of the experimental data. Although there is a small decrease in the value of $\chi^{2} / \nu$ going from LT to SLT, nevertheless, as discussed in Sec. IV B, we cannot favor one variant over the other on the basis of this value.

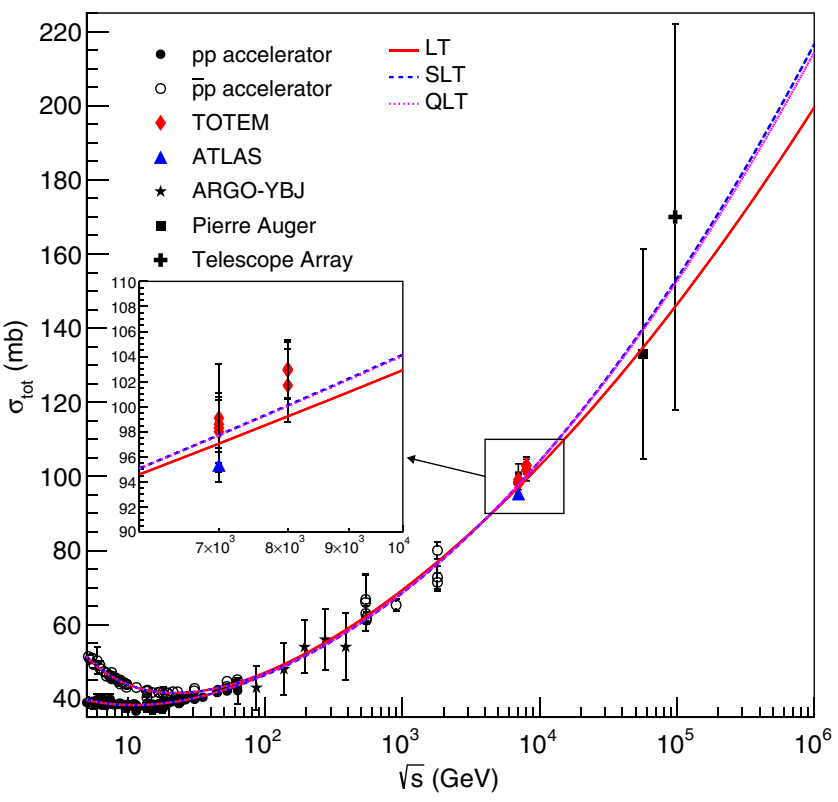

FIG. 2. Results of fits with LT, SLT and QLT to $p p$ and $\bar{p} p$ data.
Given that both are $\lesssim 1$, we can say that both variants result in good fits to the data.

Interestingly enough, the LT fit yields for $B$ a value close to the theoretical prediction $B_{\text {th }}$ obtained in Ref. [15] using the mass and spin of the $\Omega^{ \pm}$baryon.

With SLT we have obtained $C<0$ and $C \neq 0$ within the uncertainty. The negative value of $C$ causes an increase in the $B$ parameter and also in $A_{\mathbb{P}}$ to compensate for the negative contribution of the subleading term. The uncertainty on $A_{\mathbb{P}}$ increases one order of magnitude, but the relative uncertainty is still small $(\sim 5.6 \%)$.

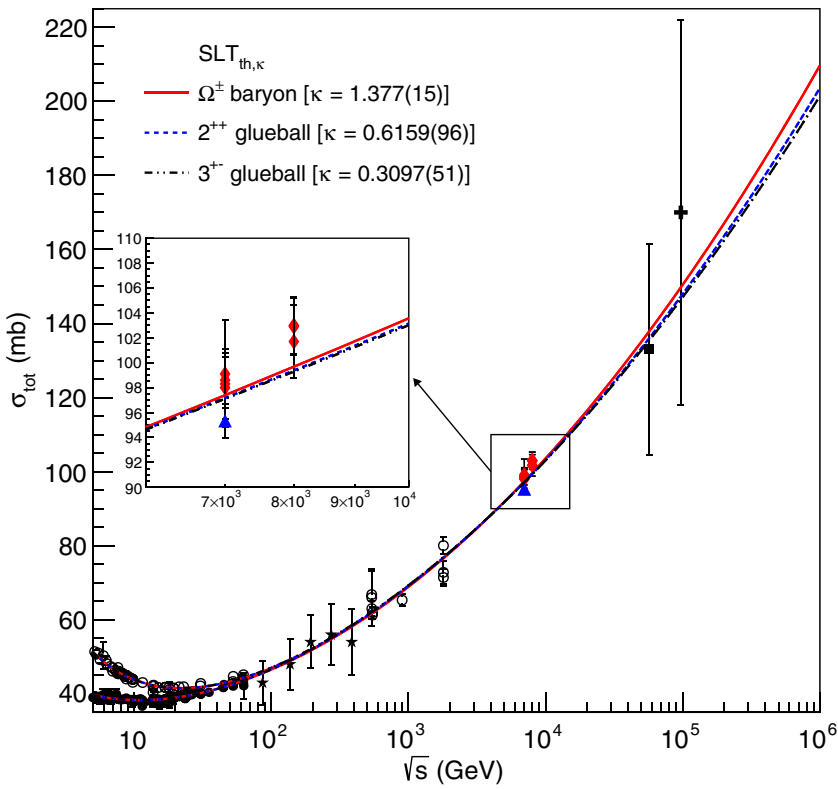

FIG. 3. Results of fits with $\mathrm{SLT}_{\mathrm{th}, \kappa}\left(\kappa\right.$ free, $B=B_{\mathrm{th}}, C=C_{\mathrm{th}}$, $Q=0$ ) to $p p$ and $\bar{p} p$ data for $B_{\text {th }}$ and $C_{\text {th }}$ calculated from the $\Omega^{ \pm}$ baryon, and the $2^{++}$and $3^{+-}$glueball states. The legend of data is the same of Fig. 2. 


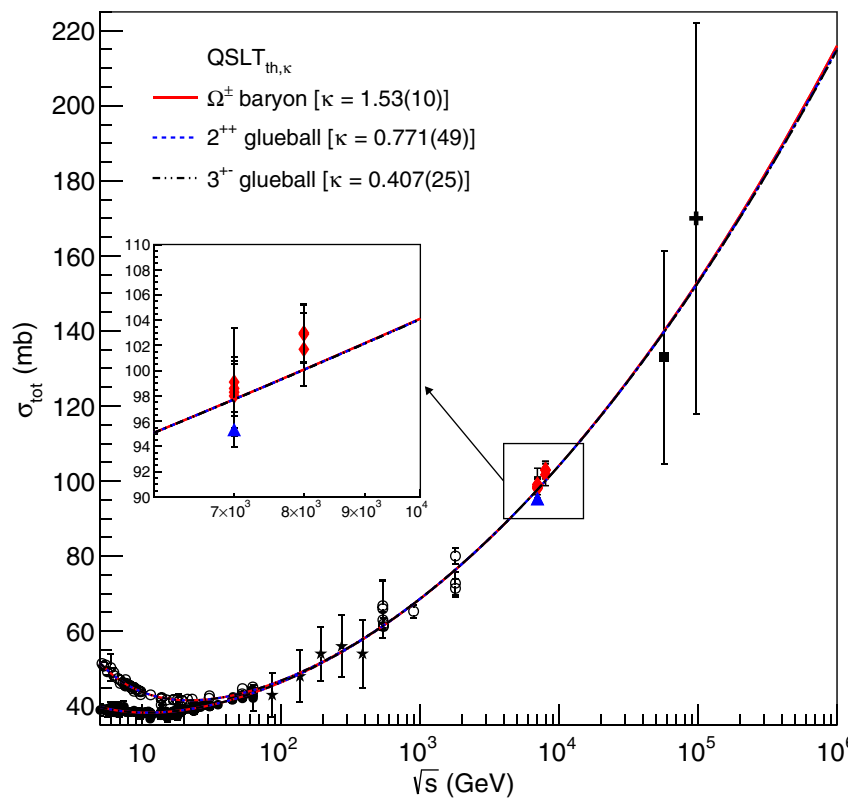

FIG. 4. Results of fits with $\operatorname{QSLT}_{\mathrm{th}, \kappa}\left(\kappa\right.$ free, $B=B_{\mathrm{th}}, C=C_{\mathrm{th}}$, $Q$ free) to $p p$ and $\bar{p} p$ data for $B_{\text {th }}$ and $C_{\text {th }}$ calculated from the $\Omega^{ \pm}$ baryon, and the $2^{++}$and $3^{+-}$glueball states. The legend of data is the same of Fig. 2.

Given the small difference mentioned above between the values of $\chi^{2} / \nu$ obtained with LT and SLT, we cannot claim that the fit with the subleading term represents an improvement over the fit without it. We can only say that the data are compatible with a nonzero value of $C$.

Regarding the Reggeon trajectories, the values of $b_{1}$ and $b_{2}$ obtained in LT are not far from the values obtained by the PDG and also in other analyses (such as, for example, the one by Menon and Silva in Ref. [52]). When changing from LT to SLT, i.e., allowing $C$ to be nonzero, we find that $A_{2}$ and $b_{2}$ are practically stable, while $A_{1}$ and $b_{1}$ increase; moreover, we observe that the values of $b_{1}$ and $b_{2}$ are compatible within the errors, i.e., the intercepts of the two Reggeon trajectories become degenerate. A similar effect was observed by COMPETE in Ref. [44], when discussing their highest-rank result (similar to LT). In that case, the degeneracy of the Reggeon intercepts was ascribed to a decreasing contribution of the $\log$-squared term for $s<s_{h}$, where $s_{h}$ is the energy scale determined in the fit.

2. Fits with $\mathrm{LT}_{\text {th }}\left(\kappa=1, B=B_{\text {th }}, C=0, Q=0\right)$, $\mathrm{SLT}_{\text {th }}$ $\left(\kappa=1, B=B_{\mathrm{th}}, C=C_{\mathrm{th}}, Q=0\right)$ and $\mathrm{SLT}_{\mathrm{th}, \kappa}(\kappa$ free,

$$
B=B_{\mathrm{th}}, C=C_{\mathrm{th}}, Q=0 \text { ) }
$$

After performing the fits with LT and SLT, we did fits where $B$ and $C$ were fixed to the values discussed in Sec. II. In Table IV we divide the results according to the variants and to the values of $B$ and $C$ that we used.

Fits with $\mathrm{LT}_{\text {th }}$ and $\mathrm{SLT}_{\mathrm{th}}: B$ and $C$ from the $\Omega^{ \pm}$baryon.The values of $B$ and $C$ calculated with the mass and spin of the $\Omega^{ \pm}$baryon [Eq. (12)] are $B_{\text {th }}=0.22 \mathrm{mb}$ and $C_{\text {th }}=-0.44 \mathrm{mb}$. In $\mathrm{LT}_{\text {th }}$ we have $B=B_{\text {th }}$ and $C=0$ fixed. The result obtained (second column of Table IV) is close to the one obtained using LT. This is not surprising, given that in that case, (see Table III) one finds $B=0.2269 \mathrm{mb}-0.23 \mathrm{mb}$, close to $B_{\mathrm{th}}$, as we have already remarked. The other parameters and the value of $\chi^{2} / \nu$ present small variations compared to LT.

On the other hand, in $\mathrm{SLT}_{\text {th }}$, where now $C=C_{\mathrm{th}}$ is fixed, the result is not satisfactory as with the previous variant. In fact, the value of $\chi^{2} / \nu$ is considerably high, indicating a poor description of the data. We would like to point out that we have practically no change in the parameters associated with the odd signature Reggeon contribution, while the intercept of the even trajectory increases going from $\mathrm{LT}_{\mathrm{th}}$ to $\mathrm{SLT}_{\text {th}}$. The same happens to $A_{1}$.

Fits with $\mathrm{LT}_{\text {th }}$ and $\mathrm{SLT}_{\mathrm{th}}: B$ and $C$ from the $2^{++}$glueball state.-In this case, we consider the (quenched) mass and spin of the $2^{++}$glueball state, giving $B_{\mathrm{th}}=-C_{\mathrm{th}}=$ $0.42 \mathrm{mb}$ [Eq. (13)]. With both variants we get a poor description of the data with $\chi^{2} / \nu \sim 3$. Besides this, the fits present a non-positive-definite error matrix. In this case, although the fit has converged, we cannot fully trust the uncertainties estimated for the free parameters [49], and so the results of our fit in general. For these reasons we have decided not to show the results in Table IV. For the sake of completeness, we mention that the intercept $b_{1}$ of the even Reggeon trajectory is very small compared to that obtained in the other cases and that we have a negative "constant" Pomeron contribution $\left(A_{\mathbb{P}}<0\right)$.

The description of the data are similar for the two variants, with overestimation of $\sigma_{\text {tot }}$ at LHC energies.

Fits with $\mathrm{LT}_{\text {th }}$ and $\mathrm{SLT}_{\mathrm{th}}$ : $B$ and $C$ from the $3^{+-}$glueball state.-Considering the $3^{+-}$glueball state, we have (using again the quenched mass) $B_{\text {th }}=0.78 \mathrm{mb}$ and $C_{\text {th }}=$ $-0.39 \mathrm{mb}$ [see Eq. (14)]. In these cases, the minimizer did not converge and, therefore, no fit results were obtained.

Fits with $\mathrm{SLT}_{\mathrm{th}, \kappa}\left(\kappa\right.$ free, $\left.B=B_{\mathrm{th}}, C=C_{\mathrm{th}}, Q=0\right)$. - In these variants we consider both $B$ and $C$ fixed with $\kappa$ free. The parameters obtained with these fits are shown in Table IV. With these variants we can estimate which of the scenarios discussed after Eq. (16) is realized at asymptotically high energies, since according to Eq. (16) the asymptotic ratio between $\sigma_{\mathrm{el}}$ and $\sigma_{\mathrm{tot}}$ is given by $\kappa / 2$.

In all the cases considered for the $B_{\text {th }}$ and $C_{\text {th }}$ values, the fits are of good quality $\left(\chi^{2} / \nu \lesssim 1\right)$ with small differences in the $\chi^{2}$ value among them. We see small variations of some parameters, for instance, $A_{1}, b_{1}$ and $A_{\mathbb{P}}$. Apart from these differences, the quality of the matching between the fitted curve and the experimental data is the same in the fitted energy range for all cases. Indeed, as can be seen in the 
inset of Fig. 3, the difference at LHC energies is very small, and the results start to be appreciably different only at cosmic-ray energies.

Regarding the value of $\kappa$, using the values of $B_{\mathrm{th}}$ and $C_{\mathrm{th}}$ obtained from the $\Omega^{ \pm}$baryon, we get $\kappa>1$, therefore an antishadowing scenario. For the glueball cases, we get $\kappa<1$; hence a grey-disk scenario, the value obtained with the $2^{++}$ glueball being larger than that obtained with the $3^{+-}$.

\section{Fits with the inclusion of the logarithmic term}

In this section, we discuss the effects of including the $\operatorname{logarithmic}$ term $(Q$ as a free parameter) in the fits discussed above. As can be seen in Table III and in Fig. 2, the logarithmic term (QLT) describes the data in the same way as SLT, i.e., with the same $\chi^{2} / \nu$. The case where both subleading terms are present (QSLT) did not converge when considering only $p p$ and $\bar{p} p$ data. Given that the $\ln s$ term and the $\ln s \cdot \ln \ln s$ term describe the data in similar ways, we attribute the nonconvergence to a competition between these two contributions. Instead, regarding the fits with $B$ and/or $C$ fixed (see Table V), we observe the same behavior seen in the fits without the logarithmic term. It is worth to mention an improvement in the $\chi^{2} / \nu$ value in $\mathrm{QSLT}_{\text {th }}$, when compared to $\mathrm{SLT}_{\text {th }}$, in the $\Omega^{ \pm}$case. We also see small improvements in the $\chi^{2}$ value for QSLT $_{\text {th }, \kappa}$ for the glueball cases and, moreover, we notice that the inclusion of the logarithmic term in QSLT $_{\text {th }, \kappa}$ leads to Reggeon trajectories which are degenerate within the errors. In all cases, the central value of the $\kappa$ parameter increases when the logarithmic contribution is present. The description of the data is the same for all the theoretical values considered.

\section{B. Fits to all reactions}

In this section we present and discuss the results obtained from fits to the full data set (data from meson-baryon and baryon-baryon scattering) with the variants LT, SLT, QLT, QSLT (Table VI), with the variants $\mathrm{LT}_{\mathrm{th}}, \mathrm{SLT}_{\mathrm{th}}$ and $\mathrm{SLT}_{\mathrm{th}, \kappa}$ (Table VII) and with the variants QLT $_{\text {th }}$, QSLT $_{\text {th }}$ and QSLT $_{\text {th }, \kappa}$ (Table VIII). The comparison of the curves of $\mathrm{SLT}_{\mathrm{th}, \kappa}$ and QSLT $_{\mathrm{th}, \kappa}$ with the experimental data is shown in Figs. 5 and 6, respectively.

\section{Fits with LT and SLT}

The results are presented in Table VI. With the LT variant we obtain a good description of the data with $\chi^{2} / \nu \sim 1$. The same is true for SLT, that we now discuss in some detail.

As already mentioned above in Sec. IV B, the SLT fit requires some care. Following the same scheme for the choice of initial values as in the analysis of $p p$ and $\bar{p} p$ data (see Fig. 1), the resulting fit has a non-positive-definite error matrix. We therefore decided to use the values obtained for the parameters $B$ and $C$ in the fit to $p p$ and $\bar{p} p$ data with the SLT (Table III) instead of the values obtained in the LT fit to all reactions. Namely, we used as
TABLE VI. Results of fits with LT, SLT, QLT and QSLT to $\sigma_{\text {tot }}$ (all data). For the units of measurement of the parameters, see Table III.

\begin{tabular}{lcccc}
\hline \hline & LT & SLT & QLT & QSLT \\
\hline$B$ & $0.2433(46)$ & $0.2652(96)$ & $0.1646(73)$ & $0.363(28)$ \\
$C$ & 0 (fixed) & $-0.200(44)$ & 0 (fixed) & $-1.32(16)$ \\
$\kappa$ & 1 (fixed) & 1 (fixed) & 1 (fixed) & 1 (fixed) \\
$b_{1}$ & $0.222(11)$ & $0.2420(85)$ & $0.2536(92)$ & $0.545(79)$ \\
$b_{2}$ & $0.5128(99)$ & $0.513(11)$ & $0.530(11)$ & $0.532(11)$ \\
$A_{1}^{p p}$ & $47.86(62)$ & $44.33(91)$ & $69.8(1.7)$ & $64.8(1.7)$ \\
$A_{2}^{p p}$ & $30.8(1.4)$ & $30.8(1.5)$ & $33.73(1.7)$ & $34.2(1.7)$ \\
$A_{\mathbb{P}}^{p p}$ & $19.0(1.1)$ & $22.61(22)$ & $6.5(1.3)$ & $33.2(2.6)$ \\
$Q^{p p}$ & 0 (fixed) & 0 (fixed) & $2.10(15)$ & $0.94(17)$ \\
$A_{1}^{p n}$ & $47.2(1.1)$ & $43.6(1.3)$ & $43.7(5.8)$ & $33.2(7.3)$ \\
$A_{2}^{p n}$ & $27.4(1.5)$ & $27.5(1.6)$ & $29.6(1.8)$ & $29.9(1.8)$ \\
$A_{\mathbb{P}}^{p n}$ & $19.2(1.1)$ & $22.86(36)$ & $22.1(3.8)$ & $40.2(2.5)$ \\
$Q^{p n}$ & 0 (fixed) & 0 (fixed) & $0.48(41)$ & $0.025(0.24)$ \\
$A_{1}^{\pi p}$ & $70.37(99)$ & $67.9(1.7)$ & $63.3(2.3)$ & $69(13)$ \\
$A_{2}^{\pi p}$ & $15.7(1.0)$ & $15.8(1.1)$ & $16.6(1.2)$ & $16.9(1.2)$ \\
$A_{\mathbb{P}}^{\pi p}$ & $-3.3(1.3)$ & $0.80(31)$ & $1.4(1.1)$ & $24.3(2.1)$ \\
$Q^{\pi p}$ & 0 (fixed) & 0 (fixed) & $0.49(12)$ & $-0.28(10)$ \\
$A_{1}^{K p}$ & $3.42(57)$ & $30.31(73)$ & $26.3(2.2)$ & $16.8(2.4)$ \\
$A_{2}^{K p}$ & $17.54(91)$ & $17.56(96)$ & $18.9(1.1)$ & $19.1(1.1)$ \\
$A_{\mathbb{P}}^{K p}$ & $1.77(85)$ & $5.09(11)$ & $6.1(1.1)$ & $17.13(92)$ \\
$Q^{K p}$ & 0 (fixed) & 0 (fixed) & $0.31(13)$ & $0.496(79)$ \\
$A_{1}^{K n}$ & $32.72(73)$ & $28.76(77)$ & $16.8(1.3)$ & $7.4(4.4)$ \\
$A_{2}^{K n}$ & $9.28(69)$ & $9.30(71)$ & $10.15(77)$ & $10.28(79)$ \\
$A_{\mathbb{N}}^{K n}$ & $1.93(84)$ & $5.22(14)$ & $10.81(70)$ & $18.1(1.0)$ \\
$Q^{K n}$ & 0 (fixed) & 0 (fixed) & $-0.159(33)$ & $0.35(11)$ \\
$\chi^{2} / \nu$ & 1.060 & 1.063 & 0.791 & 0.766 \\
$\nu$ & 532 & 531 & 527 & 526 \\
\hline \hline
\end{tabular}

initial values $B=0.349 \mathrm{mb}$ and $C=-0.95 \mathrm{mb}$ instead of $B=0.2433 \mathrm{mb}$ and $C=0$. For the other parameters the initial values remain unchanged, i.e., we used the values obtained in the LT fit to all data. With this choice we obtained a more reliable result with an accurate error matrix. In the SLT fit to all data we find again $C<0$, but with a smaller magnitude and uncertainty than in the SLT fit to $p p$ and $\bar{p} p$ data, although the relative uncertainty is the same $(\sim 22 \%)$. We attribute this to the presence of more data at low energies. On the other hand, the $\chi^{2} / \nu$ is practically the same. It is important to mention that here we are increasing the effect of low-energy data in the estimation of $C$ compared to the $p p / \bar{p} p$ fits, since we have more low-energy than high-energy data points in the present data set. In fact, we have nonzero correlation coefficients between low- and high-energy parameters, indicating the influence of the low-energy data in the determination of $C$ in the fit (see also Table 6 in Ref. [53]).

Apart from these general aspects of the fits, there is still one point that demands some comments. This point concerns the negative value of the parameter $A_{\mathbb{P}}^{\pi p}$ that 
TABLE VII. Results of fits with $\mathrm{LT}_{\text {th }}\left(\kappa=1, B=B_{\text {th }}, C=0\right.$, $Q=0), \operatorname{SLT}_{\text {th }}\left(\kappa=1, B=B_{\text {th }}, C=C_{\text {th }}, Q=0\right)$ and $\operatorname{SLT}_{\text {th }, \kappa}(\kappa$ free, $\left.B=B_{\text {th }}, C=C_{\text {th }}, Q=0\right)$ to $\sigma_{\text {tot }}$ (all data). The values of $B$ and $C$ are fixed to the theoretical values calculated with the masses and the spins of the $\Omega^{ \pm}$baryon, the $2^{++}$glueball state and the $3^{+-}$glueball state (quenched values), while the parameters $Q^{a b}$ are fixed to zero. For the units of measurement of the parameters, see Table III.

\begin{tabular}{|c|c|c|c|c|c|}
\hline & \multicolumn{3}{|c|}{$\Omega^{ \pm}$baryon } & \multirow{2}{*}{$\begin{array}{c}\begin{array}{c}2^{++} \\
\text {glueball }\end{array} \\
\mathrm{SLT}_{\mathrm{th}, \kappa}\end{array}$} & \multirow{2}{*}{$\begin{array}{c}\begin{array}{c}3^{+-} \\
\text {glueball }\end{array} \\
\mathrm{SLT}_{\mathrm{th}, \kappa}\end{array}$} \\
\hline & $\mathrm{LT}_{\text {th }}$ & $\mathrm{SLT}_{\text {th }}$ & $\mathrm{SLT}_{\mathrm{th}, \kappa}$ & & \\
\hline$B_{\text {th }}$ & $\begin{array}{c}0.22 \\
\text { (fixed) }\end{array}$ & $\begin{array}{c}0.22 \\
\text { (fixed) }\end{array}$ & $\begin{array}{c}0.22 \\
\text { (fixed) }\end{array}$ & $\begin{array}{c}0.42 \\
\text { (fixed) }\end{array}$ & $\begin{array}{c}0.78 \\
\text { (fixed) }\end{array}$ \\
\hline$C_{\text {th }}$ & $\begin{array}{c}0 \\
\text { (fixed) }\end{array}$ & $\begin{array}{l}-0.44 \\
\text { (fixed) }\end{array}$ & $\begin{array}{l}-0.44 \\
\text { (fixed) }\end{array}$ & $\begin{array}{l}-0.42 \\
\text { (fixed) }\end{array}$ & $\begin{array}{l}-0.39 \\
\text { (fixed) }\end{array}$ \\
\hline$\kappa$ & 1 (fixed) & 1 (fixed) & $1.439(23)$ & $0.653(12)$ & $0.3303(64)$ \\
\hline$Q$ & 0 (fixed) & 0 (fixed) & 0 (fixed) & 0 (fixed) & 0 (fixed) \\
\hline$\widetilde{b}_{1}$ & $0.2744(66)$ & $0.554(13)$ & $0.292(14)$ & $0.249(13)$ & $0.234(12)$ \\
\hline$b_{2}$ & $0.5141(97)$ & $0.515(11)$ & $0.514(10)$ & $0.513(11)$ & $0.513(11)$ \\
\hline$A_{1}^{p p}$ & $47.04(71)$ & $59.0(2.7)$ & $37.99(87)$ & $43.12(57)$ & $45.54(58)$ \\
\hline$A_{2}^{p p}$ & $31.0(1.4)$ & $31.4(1.6)$ & $30.9(1.4)$ & $30.8(1.5)$ & $30.8(1.5)$ \\
\hline$A_{\mathbb{P}}^{p p}$ & $23.40(24)$ & $35.159(77)$ & $29.22(51)$ & $23.76(82)$ & $21.29(98)$ \\
\hline$A_{1}^{p n}$ & $46.3(1.2)$ & $57.8(3.2)$ & $37.3(1.2)$ & $42.4(1.1)$ & $44.9(1.1)$ \\
\hline$A_{2}^{p n}$ & $27.6(1.5)$ & $27.9(1.7)$ & $27.5(1.6)$ & $27.5(1.6)$ & $27.4(1.6)$ \\
\hline$A_{\mathbb{P}}^{p n}$ & 23.64(37) & $35.24(15)$ & $29.44(56)$ & $24.01(87)$ & $21.5(1.0)$ \\
\hline$A_{1}^{\pi p}$ & $73.5(1.5)$ & $136.9(8.2)$ & $64.8(2.3)$ & $67.0(1.5)$ & $68.6(1.2)$ \\
\hline$A_{2}^{\pi p}$ & $16.11(98)$ & $16.7(1.1)$ & $16.1(1.0)$ & $15.9(1.1)$ & $15.8(1.1)$ \\
\hline$A_{\mathbb{P}}^{\pi p}$ & $19.84(28)$ & $15.921(83)$ & $8.27(65)$ & $20.8(1.0)$ & $-0.69(1.2)$ \\
\hline$A_{1}^{K p}$ & $32.53(51)$ & 28.1(1.4) & $22.61(49)$ & $28.95(47)$ & $31.68(54)$ \\
\hline$A_{2}^{K p}$ & $17.67(88)$ & 17.66(98) & $17.57(92)$ & $17.55(96)$ & 17.54(97) \\
\hline$A_{\mathbb{P}}^{K p}$ & $5.39(18)$ & $15.450(48)$ & $11.09(37)$ & $6.15(64)$ & $3.90(78)$ \\
\hline$A_{1}^{K n}$ & $30.89(72)$ & $23.8(1.8)$ & $20.86(64)$ & $27.38(71)$ & $30.16(72)$ \\
\hline$A_{2}^{K n}$ & $9.35(69)$ & $9.43(73)$ & $9.33(70)$ & $9.30(71)$ & $9.29(72)$ \\
\hline$A_{\mathbb{P}}^{K n}$ & $5.48(20)$ & $15.388(71)$ & $11.18(36)$ & $6.27(64)$ & $4.04(77)$ \\
\hline$\chi^{2} / \nu$ & 1.108 & 1.966 & 1.071 & 1.062 & 1.061 \\
\hline$\nu$ & 533 & 533 & 532 & 532 & 532 \\
\hline
\end{tabular}

appears in LT, while it changes to a positive value in SLT. This is the only negative constant Pomeron contribution (although with large errors) in this set of fits. Taking into account the property of factorization of the residues of the Regge poles [8] (see also the comments in Ref. [44]), this parameter is expected to be positive. However, factorization is only proven in the case of simple poles, and it is valid when only one trajectory dominates [8]. As already commented in footnote 4 in Sec. III, $A_{\mathbb{P}}^{a b}$ could also originate from the sum of a constant (simple-pole) Pomeron with the constant term coming from higher-order singularities (e.g., a triple-pole Pomeron) and, in any case (see also the discussion at the end of Sec. III), its value is affected by the choice of the energy scale in the leading and subleading terms in Eq. (20). Therefore, we cannot exclude this result only on the basis of factorization. On the other hand, even with $A_{\mathbb{P}}^{\pi p}<0$, we do not have a negative
TABLE VIII. Results of fits with $\mathrm{QLT}_{\text {th }}\left(\kappa=1, B=B_{\text {th }}\right.$, $C=0, Q$ free), $\mathrm{QSLT}_{\text {th }}\left(\kappa=1, B=B_{\mathrm{th}}, C=C_{\mathrm{th}}, Q\right.$ free $)$ and $\mathrm{QSLT}_{\text {th }, \kappa}\left(\kappa\right.$ free, $B=B_{\mathrm{th}}, C=C_{\mathrm{th}}, Q$ free) to $\sigma_{\mathrm{tot}}$ (all data). The values of $B$ and $C$ are fixed to the theoretical values calculated with the masses and the spins of the $\Omega^{ \pm}$baryon, the $2^{++}$glueball state and the $3^{+-}$glueball state (quenched values). For the units of measurement of the parameters, see Table III.

\begin{tabular}{|c|c|c|c|c|c|}
\hline & \multicolumn{3}{|c|}{$\Omega^{ \pm}$baryon } & \multirow{2}{*}{$\begin{array}{c}\begin{array}{c}2^{++} \\
\text {glueball }\end{array} \\
\text { QSLT }_{\text {th }, \kappa}\end{array}$} & \multirow{2}{*}{$\begin{array}{c}\begin{array}{c}3^{+-} \\
\text {glueball }\end{array} \\
\text { QSLT }_{\text {th }, \kappa}\end{array}$} \\
\hline & $\mathrm{QLT}_{\mathrm{th}}$ & $\mathrm{QSLT}_{\mathrm{th}}$ & $\mathrm{QSLT}_{\mathrm{th}, \kappa}$ & & \\
\hline$B_{\text {th }}$ & $\begin{array}{c}0.22 \\
\text { (fixed) }\end{array}$ & $\begin{array}{c}0.22 \\
\text { (fixed) }\end{array}$ & $\begin{array}{c}0.22 \\
\text { (fixed) }\end{array}$ & $\begin{array}{c}0.42 \\
\text { (fixed) }\end{array}$ & $\begin{array}{c}0.78 \\
\text { (fixed) }\end{array}$ \\
\hline$C_{\mathrm{th}}$ & $\begin{array}{c}0 \\
\text { (fixed) }\end{array}$ & $\begin{array}{l}-0.44 \\
\text { (fixed) }\end{array}$ & $\begin{array}{l}-0.44 \\
\text { (fixed) }\end{array}$ & $\begin{array}{l}-0.42 \\
\text { (fixed) }\end{array}$ & $\begin{array}{l}-0.39 \\
\text { (fixed) }\end{array}$ \\
\hline$\kappa$ & 1 (fixed) & 1 (fixed) & $1.54(11)$ & $0.774(51)$ & $0.408(23)$ \\
\hline$b_{1}$ & $0.331(20)$ & $0.307(18)$ & $0.565(93)$ & $0.576(92)$ & $0.582(80)$ \\
\hline$b_{2}$ & $0.531(11)$ & $0.531(11)$ & $0.532(11)$ & $0.532(11)$ & $0.532(11)$ \\
\hline$A_{1}^{p p}$ & $57.5(1.4)$ & $63.0(1.2)$ & $62.3(8.7)$ & $61.0(8.8)$ & $60.4(7.8)$ \\
\hline$A_{2}^{p p}$ & $33.9(1.7)$ & $33.9(1.7)$ & $34.2(1.8)$ & $34.2(1.8)$ & $34.2(1.8)$ \\
\hline$A_{\mathbb{P}}^{p p}$ & $23.2(1.5)$ & $15.8(1.8)$ & $37.5(3.3)$ & $39.8(3.1)$ & $40.9(2.7)$ \\
\hline$Q^{p p}$ & $0.20(12)$ & $1.88(13)$ & $-0.71(46)$ & $-1.61(47)$ & $-2.03(42)$ \\
\hline$A_{1}^{p n}$ & $32.9(8.8)$ & $38.3(9.0)$ & $30.4(9.8)$ & $29(10)$ & 28(10) \\
\hline$A_{2}^{p n}$ & 29.7(1.8) & 29.7(1.8) & 29.9(1.8) & $29.9(1.8)$ & $29.9(1.8)$ \\
\hline$A_{\mathbb{P}}^{p^{n}}$ & $34.7(5.4)$ & $28.3(5.0)$ & $44.0(3.2)$ & $46.1(3.0)$ & $47.1(2.7)$ \\
\hline$Q^{p n}$ & $-1.11(53)$ & $0.49(57)$ & $-1.57(47)$ & $-2.45(47)$ & $-2.86(44)$ \\
\hline$A_{1}^{\pi p}$ & $52.7(3.1)$ & $57.7(2.6)$ & $66.7(1.5)$ & $65.6(1.5)$ & $65(13)$ \\
\hline$A_{2}^{\pi p}$ & $16.7(1.2)$ & $16.7(1.2)$ & $16.9(1.2)$ & $16.8(1.2)$ & $16.8(1.2)$ \\
\hline$A_{\mathbb{P}}^{\pi p}$ & $16.6(1.7)$ & $9.6(2.0)$ & $28.6(2.8)$ & $30.8(2.7)$ & $31.9(2.3)$ \\
\hline$Q^{\pi p}$ & $-1.28(15)$ & $0.36(16)$ & $-1.94(40)$ & $-2.84(42)$ & $-3.26(38)$ \\
\hline$A_{1}^{K p}$ & $17.0(2.7)$ & $21.9(1.2)$ & $12.7(3.5)$ & $10.4(3.6)$ & $9.3(3.6)$ \\
\hline$A_{2}^{K p}$ & $19.0(1.1)$ & $19.0(1.1)$ & 19.1(1.1) & 19.1(1.1) & 19.1(1.1) \\
\hline$A_{\mathbb{P}}^{K p}$ & $15.6(1.4)$ & 10.10(99) & 21.0(1.6) & $23.0(1.5)$ & $24.0(1.3)$ \\
\hline$Q^{K p}$ & $-1.04(15)$ & $0.481(91)$ & $-1.11(27)$ & $-1.98(30)$ & $-2.39(27)$ \\
\hline$A_{1}^{K n}$ & $8.7(5.1)$ & $13.5(5.2)$ & $25.6(6.7)$ & $0.036(7.1)$ & $-1.2(6.9)$ \\
\hline$A_{2}^{K n}$ & $10.21(81)$ & $10.20(80)$ & $10.28(81)$ & $10.27(81)$ & $10.27(81)$ \\
\hline$A_{\mathbb{P}}^{K n}$ & $18.5(2.3)$ & $13.4(2.6)$ & $22.0(1.6)$ & $24.0(1.5)$ & $24.9(1.4)$ \\
\hline$Q^{K n}$ & $-1.36(27)$ & $0.13(29)$ & $-1.25(27)$ & $-2.12(29)$ & $-2.53(27)$ \\
\hline$\chi^{2} / \nu$ & 0.778 & 0.781 & 0.764 & 0.764 & 0.764 \\
\hline r & 528 & 528 & 527 & 527 & 527 \\
\hline
\end{tabular}

Pomeron contribution (from the combination of this constant term plus the $\ln ^{2} s$ term) in LT.

\section{Fits with $\mathrm{LT}_{\mathrm{th}}\left(\kappa=1, B=B_{\mathrm{th}}, C=0, Q=0\right)$, $\mathrm{SLT} \mathrm{T}_{\mathrm{th}}$ $\left(\kappa=1, B=B_{\mathrm{th}}, C=C_{\mathrm{th}}, Q=0\right)$ and $\mathrm{SLT}_{\mathrm{th}, \kappa}(\kappa$ free, $B=B_{\mathrm{th}}, C=C_{\mathrm{th}}, Q=0$ )}

In Table VII we present the results obtained with the variants $\mathrm{LT}_{\text {th }}$ and $\mathrm{SLT}_{\text {th }}$, where $B$ and $C$ are fixed to their theoretical values [see Eqs. (12)-(14)].

Fits with $\mathrm{LT}_{\text {th }}$ and $\mathrm{SLT}_{\mathrm{th}}: B$ and $C$ from the $\Omega^{ \pm}$baryon.The results (second and third column of Table VII) obtained in this case are satisfactory for $\mathrm{LT}_{\text {th }}$ with only a small increase of $\chi^{2} / \nu$ in comparison with LT. For $\mathrm{SLT}_{\text {th }}$, we get 

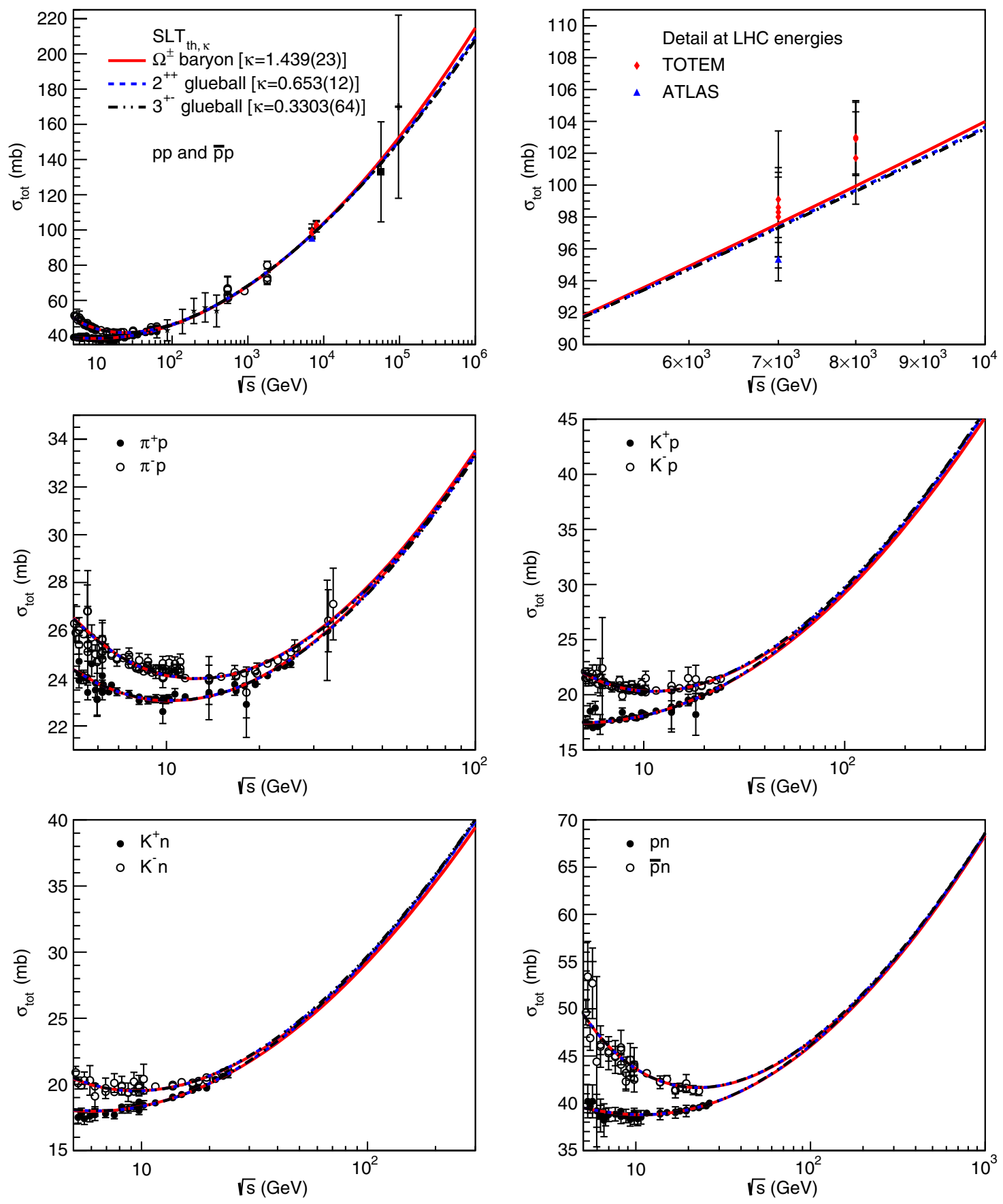

FIG. 5. Results of fits with $\operatorname{SLT}_{\text {th }, \kappa}\left(\kappa\right.$ free, $B=B_{\text {th }}, C=C_{\text {th }}, Q=0$ ) to all data for $B_{\text {th }}$ and $C_{\text {th }}$ calculated from the $\Omega^{ \pm}$baryon, and the $2^{++}$and $3^{+-}$glueball states. The legend for the curves is shown in the top-left panel. For the legend of $p p$ and $\bar{p} p$ data see Fig. 2.

$\chi^{2} / \nu \sim 2$ while in SLT we have $\sim 1$. However, this increase is less than that observed in the fits to $p p$ and $\bar{p} p$ data only. Contrary to the LT fit, we have that all $A_{\mathbb{P}}^{i}>0$ in $\mathrm{LT}_{\text {th }}$.

Fits with $\mathrm{LT}_{\mathrm{th}}$ and $\mathrm{SLT}_{\mathrm{th}}: B$ and $C$ from the $2^{++}$glueball state. - The fits with $\mathrm{LT}_{\text {th }}$ and $\mathrm{SLT}_{\text {th }}$ in this case have a nonpositive-definite error matrix. The results (not presented in Table VII) have the same features of the fits to $p p$ and $\bar{p} p$ data, for example, a very small $b_{1}$ parameter. We also have that almost all $A_{\mathbb{P}}^{i}<0$. The $\chi^{2} / \nu$ values are around 1.5 , with a similar quality in the matching with data for both variants. Regarding $p p$ and $\bar{p} p$, the fits overestimate the data at LHC energies, reaching the upper error bar of the TOTEM data.

Fits with $\mathrm{LT}_{\text {th }}$ and $\mathrm{SLT}_{\mathrm{th}}$ : $B$ and $C$ from the $3^{+-}$glueball state.-Again, using the mass and spin of the $3^{+-}$glueball state, the fits did not converge. 

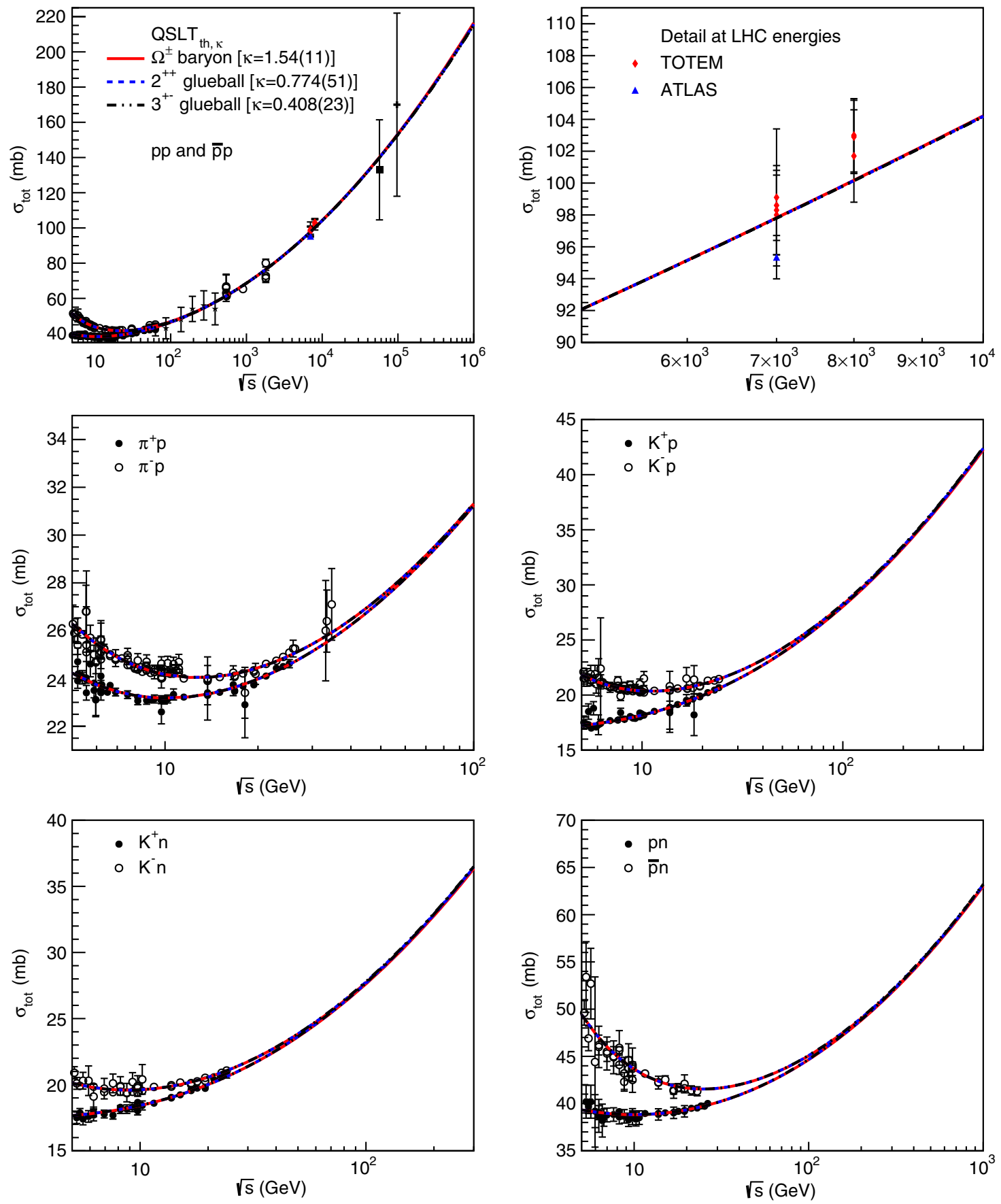

FIG. 6. Results of fits with QSLT $\mathrm{Qth}_{\kappa}$ ( $\kappa$ free, $B=B_{\mathrm{th}}, C=C_{\mathrm{th}}, Q$ free) to all data for $B_{\mathrm{th}}$ and $C_{\mathrm{th}}$ calculated from the $\Omega^{ \pm}$baryon, and the $2^{++}$and $3^{+-}$glueball states. The legend for the curves is shown in the top-left panel. For the legend of $p p$ and $\bar{p} p$ data see Fig. 2.

Fits with $\mathrm{SLT}_{\mathrm{th}, \kappa}\left(\kappa\right.$ free, $\left.B=B_{\mathrm{th}}, C=C_{\mathrm{th}}, Q=0\right)$. - Finally we discuss the results of the fits where $\kappa$ is a free parameter. We show in Table VII all the parameters determined in the fits. The $\chi^{2} / \nu$ values are close to 1 , indicating that the fit is of good quality. Furthermore, we get a good description of data in all cases. As in the fit with SLT, the "constant" Pomeron term $A_{\mathbb{P}}^{\pi p}$ is positive, except when we consider the $3^{+-}$glueball state, in which case we find a negative central value, which is however compatible with zero.
The agreement between the fitted curve and the data (Fig. 5) is also similar to that achieved when fitting only the $p p$ and $\bar{p} p$ data: in the energy range of the fit all cases give almost indistinguishable curves, which present small differences in the extrapolation to higher energies. In conclusion, the three choices for $B_{\text {th }}$ and $C_{\text {th }}$ all give equally good fits.

Concerning the parameter $\kappa$, we have $\kappa>1$ for the $\Omega^{ \pm}$ baryon and $\kappa<1$ for the $2^{++}$and $3^{+-}$glueball states (with 
smaller $\kappa$ in the latter case), as it was also found when fitting $p p$ and $\bar{p} p$ data only, but with slightly bigger central values.

\section{Fits with inclusion of the logarithmic term}

Overall, the inclusion of the logarithmic term in the fits to all reactions decreases the value of $\chi^{2} / \nu$ from about 1 (for the SLT fit) to about 0.8 (for the QLT and QSLT fits). (Of course, some care is needed in judging the implications of this change in the $\chi^{2} / \nu$. Following the approach of Ref. [15], the coefficient of the logarithmic term, differently from $B$ and $C$, is expected to be reaction dependent. This means the inclusion of only one more free parameter when analyzing the $p p / \bar{p} p$ case, but when we consider all other reactions we have to include one free parameter for each pair of crossed channels, therefore five new free parameters.) Analyzing the values of $Q^{a b}$ (see Table VI), we see that some of them change their sign when going from QLT to QSLT. We understand that the presence of the subleading term of type $\ln s \cdot \ln \ln s$ creates some competition between the two subleading contributions. We recall that the fit QSLT to only $p p$ and $\bar{p} p$ data did not converge. Fits with $B$ and/or $C$ fixed (see Table VIII) show the same features of the fits to only $p p$ and $\bar{p} p$ data, but with a decrease of the $\chi^{2} / \nu$ values. When $\kappa$ is free, we obtain the same scenarios that have been obtained without the logarithmic term, but with a slightly larger central value for the parameter $\kappa$.

\section{CONCLUSIONS}

In this work we have performed a phenomenological analysis of total cross section data from hadronic scattering in order to estimate the relevance of a subleading $\ln \left(s / s_{0}\right) \ln \ln \left(s / s_{0}\right)$ term, obtained in recent theoretical studies in nonperturbative QCD [15] (see also Refs. [2224] for a similar subleading term). More precisely, we have used the following parametrization of the total cross section in the "high-energy" region:

$$
\begin{aligned}
\sigma_{\text {tot }}^{a b}(s) \underset{s \rightarrow \infty}{\sim} & B \ln ^{2}\left(\frac{s}{s_{0}^{a b}}\right)+C \ln \left(\frac{s}{s_{0}^{a b}}\right) \ln \left[\ln \left(\frac{s}{s_{0}^{a b}}\right)\right] \\
& +Q^{a b} \ln \left(\frac{s}{s_{0}^{a b}}\right),
\end{aligned}
$$

with $s_{0}^{a b}=m_{a} m_{b}$. The "low-energy" region has been parametrized as usual in terms of Reggeon exchange in the $t$-channel plus a constant term. We have performed best fits to two data sets with total center-of-mass energy $\sqrt{s} \geq 5 \mathrm{GeV}$ : (i) only $p p$ and $\bar{p} p$ scattering and (ii) all meson-baryon and baryon-baryon data available at the PDG website [45] (except for $\Sigma^{-} p$ ). In both cases, the recent data for $p p$ scattering obtained at 7 and $8 \mathrm{TeV}$ by the TOTEM and ATLAS Collaborations [9-14] were included in the fits.
As discussed in Sec. II, the parameters $B$ and $C$ may be written, following the nonperturbative QCD approach of Ref. [15], as $B=\kappa B_{\text {th }}$ and $C=\kappa C_{\text {th }}$, where $B_{\text {th }}$ and $C_{\text {th }}$ are written in terms of the spin $\tilde{s}$ and the mass $\tilde{m}$ of the particle that maximizes the ratio of Eq. (7) [see Eq. (10)], while the parameter $\kappa$ is associated to the asymptotic value of the ratio $\sigma_{\text {el }} / \sigma_{\text {tot }}$ [see Eq. (16)]. While in Ref. [15] $B_{\text {th }}$ and $C_{\text {th }}$ were estimated from the hadronic spectrum, here we could in principle try to reverse the approach, and, using Eq. (11), estimate $\tilde{s}$ and $\tilde{m}$ (or, better, $\tilde{m} / \sqrt{\kappa}$ ) from the values of $B$ and $C$ obtained from the fit.

In general, we have obtained a good description of the data with parametrizations that generalize the highestrank result of the COMPETE Collaboration, by including a $\ln s$ and/or a $\ln s \cdot \ln \ln s$ term besides the $\ln ^{2} s$ term. Unfortunately, as discussed in Sec. V (see, in particular, the results of the fits LT, SLT, QLT and QSLT in Tables III and VI and in Fig. 2), this type of analysis, in which $B, C$ and $Q$ are treated as free parameters, was not conclusive, because of the "competition" between the two subleading terms $\ln s$. $\ln \ln s$ and $\ln s$ in the range of energy considered. (The same conclusion was obtained also in Ref. [24], where an analysis similar to ours was performed.) In particular, the quality of the fits to $p p$ and $\bar{p} p$ data (i.e., the data set more sensitive to the high-energy behavior) with only one of the two subleading terms or without any of them are comparable, so that we cannot clearly state which one is the best. When fitting our parametrizations to all reactions, the value of $\chi^{2} / \nu$ decreases from about 1, for the SLT fit, to about 0.8, for the QLT and QSLT fits, but, again, no conclusive statement can be made.

Therefore, we have decided to restrict our analysis, considering fits where the $B$ and $C$ parameters are set to $B=\kappa B_{\text {th }}$ and $C=\kappa C_{\text {th }}$ [see Eq. (10)], with $\kappa$ treated as an extra free parameter, while $B_{\text {th }}$ and $C_{\text {th }}$ have been fixed to the values calculated for the three relevant cases which were considered and discussed in Ref. [15], i.e., the $\Omega^{ \pm}$ baryon and the $2^{++}$and $3^{+-}$glueball states [see Eqs. (12), (13) and (14)]. In Table IX we display all the resulting values for the ratio $\sigma_{\mathrm{el}} / \sigma_{\mathrm{tot}}=\kappa / 2$ and for the coefficient $B=\kappa B_{\text {th }}$. (For comparison, we also show the values of the ratio $\sigma_{\mathrm{el}} / \sigma_{\mathrm{tot}}=\kappa / 2$ calculated from the $\kappa$ values determined, using the relation $\kappa=B / B_{\text {th }}$, from fits LT and QLT to $p p / \bar{p} p$ data only and also from fits to all reactions.)

The fits with the $\Omega^{ \pm}$baryon values indicate an asymptotic scenario in the antishadowing regime, since $\sigma_{\mathrm{el}} / \sigma_{\mathrm{tot}}>0.5$. On the other hand, the values inferred from the fits with $B$ and $C$ fixed to the values obtained from the $2^{++}$glueball state indicate a grey-disk scenario, with $\sigma_{\mathrm{el}} / \sigma_{\text {tot }} \sim 0.31 \div 0.38$. This value is in agreement with the asymptotic ratio obtained in other studies

(i) empirical fits to the $\sigma_{\mathrm{el}} / \sigma_{\mathrm{tot}}$ data made by Fagundes, Menon and Silva [54];

(ii) independent fits to $\sigma_{\text {tot }}$ and $\sigma_{\text {el }}$ data also made by Fagundes, Menon and Silva [52,53,55] that yield this ratio close to 0.3 ; 
TABLE IX. Ratio $\sigma_{\mathrm{el}} / \sigma_{\text {tot }}=\kappa / 2$ and value of the coefficient $B=\kappa B_{\text {th }}$, with $\kappa$ determined from the fits $\mathrm{SLT}_{\text {th }, \kappa}$ and QSLT $_{\text {th }, \kappa}$ to $p p$ and $\bar{p} p$ data only, and also from fits to data for all reactions. For comparison, we also show the values of the ratio $\sigma_{\mathrm{el}} / \sigma_{\mathrm{tot}}=\kappa / 2$ calculated from the $\kappa$ values determined, using the relation $\kappa=B / B_{\mathrm{th}}$, from fits LT and QLT to $p p / \bar{p} p$ data only and also from fits to all reactions. The theoretical inputs for $B_{\text {th }}$ are those discussed in the text, namely the $\Omega^{ \pm}$baryon and the $2^{++}$and $3^{+-}$glueball states.

\begin{tabular}{|c|c|c|c|c|}
\hline \multirow[b]{2}{*}{$\sigma_{\mathrm{el}} / \sigma_{\mathrm{tot}}=\kappa / 2$} & \multicolumn{2}{|c|}{ Fits to $p p / \bar{p} p$ data only } & \multicolumn{2}{|c|}{ Fits to all reactions } \\
\hline & $\mathrm{SLT}_{\mathrm{th}, \kappa}$ & $\mathrm{QSLT}_{\mathrm{th}, \kappa}$ & $\mathrm{SLT}_{\mathrm{th}, \kappa}$ & $\mathrm{QSLT}_{\mathrm{th}, \kappa}$ \\
\hline$\Omega^{ \pm}$baryon & $0.6885(91)$ & $0.765(50)$ & $0.720(12)$ & $0.770(55)$ \\
\hline $2^{++}$glueball & $0.3080(48)$ & $0.385(25)$ & $0.3265(60)$ & $0.387(26)$ \\
\hline \multirow{2}{*}{$3^{+-}$glueball } & $0.1548(26)$ & $0.203(13)$ & $0.1652(32)$ & $0.204(12)$ \\
\hline & LT & QLT & LT & QLT \\
\hline$\Omega^{ \pm}$baryon & $0.5155(85)$ & $0.707(43)$ & $0.553(11)$ & $0.374(17)$ \\
\hline $2^{++}$glueball & $0.2701(45)$ & $0.370(23)$ & $0.2896(55)$ & $0.1960(87)$ \\
\hline \multirow[t]{2}{*}{$3^{+-}$glueball } & $0.1454(24)$ & $0.199(12)$ & $0.1560(30)$ & $0.1055(47)$ \\
\hline & \multicolumn{2}{|c|}{ Fits to $p p / \bar{p} p$ data only } & \multicolumn{2}{|c|}{ Fits to all reactions } \\
\hline$B=\kappa B_{\text {th }}$ & $\mathrm{SLT}_{\mathrm{th}, \kappa}$ & QSLT $_{\text {th }, \kappa}$ & $\mathrm{SLT}_{\mathrm{th}, \kappa}$ & QSLT $_{\text {th }, \kappa}$ \\
\hline$\Omega^{ \pm}$baryon & $0.303(4)$ & $0.337(22)$ & $0.317(5)$ & $0.339(24)$ \\
\hline $2^{++}$glueball & $0.259(4)$ & $0.324(21)$ & $0.274(5)$ & $0.325(21)$ \\
\hline $3^{+-}$glueball & $0.242(4)$ & $0.317(20)$ & $0.258(5)$ & $0.318(18)$ \\
\hline
\end{tabular}

(iii) the prediction made by Kohara, Ferreira, and Kodama [56], using the stochastic vacuum model and fits to elastic scattering data, that this ratio is below $1 / 2$ (and close to $1 / 3$ );

(iv) the scenario of a black torus proposed by Dremin [57-59], where the particle has a semitransparent center (grey disk) surrounded by a black disk. This scenario has also been proposed, in a different context, in Refs. [60,61].

Of course, we cannot claim that this result provides a confirmation of the other approaches. On the other hand, it is interesting that a similar value for this ratio is obtained from an analysis of the total cross section alone, provided that some theoretical input on $B_{\text {th }}$ (and $C_{\text {th }}$ ) is used.

Using the mass and spin of the $3^{+-}$glueball state, we also get a grey-disk scenario: however, the resulting asymptotic value is smaller than the experimental data available so far. From Table IX, we see that the value for this ratio is around $0.16-0.20$, while the experimental value at the highest energy obtained so far $(8 \mathrm{TeV})$ is approximately 0.27 . Since by now the data show a rising trend with energy (see for instance Fig. 1 in Ref. [54]), this means that if this scenario is the correct one, then the data must present a local maximum and then decrease as the energy increases until it reaches the asymptotic value. Although there seems to be no theoretical reason to exclude this type of behavior, it seems quite unlikely to happen, and we would rather expect a smooth rise with energy until the asymptotic value is reached.

The results in Table IX show that the inclusion of the $\ln s \ln \ln s$ term, although not changing radically the value of $\sigma_{\mathrm{el}} / \sigma_{\mathrm{tot}}$, still has an effect of the order of $10 \%$ in most of the cases.

In conclusion, high-energy data in a larger range than what is currently available will be necessary in order to be able to properly estimate the contribution of the subleading term and to discriminate between the different scenarios which have been presented above.

\section{ACKNOWLEDGMENTS}

P. V. R. G. Silva thanks the financial support by São Paulo Research Foundation (FAPESP) under the Contracts No. 2013/27060-3 and No. 2015/21855-0. The authors thank M. J. Menon for useful discussions.
[1] F. Halzen and A. D. Martin, Quarks and Leptons: An Introductory Course in Modern Particle Physics (Wiley, New York, USA, 1984), 0471887412, 9780471887416.
[2] G. Pancheri and Y. N. Srivastava, Eur. Phys. J. C 77, 150 (2017).

[3] M. Froissart, Phys. Rev. 123, 1053 (1961). 
[4] A. Martin, Phys. Rev. 129, 1432 (1963).

[5] A. Martin, Nuovo Cimento A 42, 930 (1966).

[6] L. Łukaszuk and A. Martin, Il Nuovo Cimento A 52, 122 (1967).

[7] P. D. B. Collins, An Introduction to Regge Theory and HighEnergy Physics, Cambridge Monographs on Mathematical Physics (Cambridge University Press, Cambridge, England, 2009), 9780521110358, http://www-spires.fnal.gov/spires/ find/books/www?cl=QC793.3.R4C695.

[8] V. Barone and E. Predazzi, High-Energy Particle Diffraction, Texts and Monographs in Physics Vol. 565 (SpringerVerlag, Berlin Heidelberg, 2002), 354042X1076, http:// www-spires.fnal.gov/spires/find/books/www?cl=QC794.6 .C6B37::2002.

[9] G. Antchev et al. (TOTEM Collaboration), Europhys. Lett. 96, 21002 (2011).

[10] G. Antchev et al. (TOTEM Collaboration), Europhys. Lett. 101, 21002 (2013).

[11] G. Antchev et al. (TOTEM Collaboration), Europhys. Lett. 101, 21004 (2013).

[12] G. Antchev et al. (TOTEM Collaboration), Phys. Rev. Lett. 111, 012001 (2013).

[13] G. Antchev et al. (TOTEM Collaboration), Eur. Phys. J. C 76, 661 (2016).

[14] G. Aad et al. (ATLAS Collaboration), Nucl. Phys. B889, 486 (2014).

[15] M. Giordano and E. Meggiolaro, J. High Energy Phys. 03 (2014) 002.

[16] O. Nachtmann, Ann. Phys. (N.Y.) 209, 436 (1991).

[17] H. G. Dosch, E. Ferreira, and A. Krämer, Phys. Rev. D 50, 1992 (1994).

[18] M. Rueter and H. G. Dosch, Phys. Lett. B 380, 177 (1996).

[19] O. Nachtmann, Lect. Notes Phys. 479, 49 (1997).

[20] E. R. Berger and O. Nachtmann, Eur. Phys. J. C 7, 459 (1999).

[21] A. I. Shoshi, F. D. Steffen, and H. J. Pirner, Nucl. Phys. A709, 131 (2002).

[22] A. Martin and S. M. Roy, Phys. Rev. D 89, 045015 (2014).

[23] H. Nastase and J. Sonnenschein, Phys. Rev. D 92, 105028 (2015).

[24] V. E. Díez, R. M. Godbole, and A. Sinha, Phys. Lett. B 746, 285 (2015).

[25] E. Meggiolaro, Z. Phys. C 76, 523 (1997).

[26] E. Meggiolaro, Eur. Phys. J. C 4, 101 (1998).

[27] E. Meggiolaro, Nucl. Phys. B625, 312 (2002).

[28] E. Meggiolaro, Nucl. Phys. B707, 199 (2005).

[29] M. Giordano and E. Meggiolaro, Phys. Rev. D 74, 016003 (2006).

[30] E. Meggiolaro, Phys. Lett. B 651, 177 (2007).

[31] M. Giordano and E. Meggiolaro, Phys. Lett. B 675, 123 (2009).

[32] R. M. Corless, G. H. Gonnet, D. E. Hare, D. J. Jeffrey, and D. E. Knuth, Adv. Comput. Math. 5, 329 (1996).

[33] C. J. Morningstar and M. J. Peardon, Phys. Rev. D 60, 034509 (1999).
[34] K. A. Olive et al. (Particle Data Group Collaboration), Chin. Phys. C 38, 090001 (2014).

[35] C. Patrignani et al. (Particle Data Group Collaboration), Chin. Phys. C 40, 100001 (2016).

[36] M. M. Block and R. N. Cahn, Rev. Mod. Phys. 57, 563 (1985).

[37] S. M. Troshin and N.E. Tyurin, Phys. Lett. B 316, 175 (1993).

[38] S. M. Troshin and N. E. Tyurin, Int. J. Mod. Phys. A 22, 4437 (2007).

[39] G. Antchev et al. (TOTEM Collaboration), Nucl. Phys. B899, 527 (2015).

[40] M. Aaboud et al. (ATLAS Collaboration), Phys. Lett. B 761, 158 (2016).

[41] N. A. Amos et al. (E710 Collaboration), Phys. Rev. Lett. 68, 2433 (1992).

[42] F. Abe et al. (CDF Collaboration), Phys. Rev. D 50, 5550 (1994).

[43] C. Avila et al. (E811 Collaboration), Phys. Lett. B 445, 419 (1999).

[44] J. R. Cudell, V. Ezhela, P. Gauron, K. Kang, Yu. V. Kuyanov, S. Lugovsky, B. Nicolescu, and N. Tkachenko, Phys. Rev. D 65, 074024 (2002).

[45] http://pdg.lbl.gov/2015/hadronic-xsections/hadron.html.

[46] G. Aielli et al. (ARGO-YBJ Collaboration), Phys. Rev. D 80, 092004 (2009).

[47] P. Abreu et al. (Pierre Auger Observatory Collaboration), Phys. Rev. Lett. 109, 062002 (2012).

[48] R. U. Abbasi et al. (Telescope Array Collaboration), Phys. Rev. D 92, 032007 (2015).

[49] F. James, MINUIT Function Minimization and Error Analysis: Reference Manual Version 94.1 (CERN, Geneva1994).

[50] P. Bevington and D. Robinson, Data Reduction and Error Analysis for the Physical Sciences Boston (McGraw-Hill, Massachusetts, 1992), 0079112439.

[51] http://root.cern.ch/.

[52] M. J. Menon and P. V. R. G. Silva, J. Phys. G 40, 125001 (2013); 41, 019501(E) (2014).

[53] M. J. Menon and P. V. R. G. Silva, Int. J. Mod. Phys. A 28, 1350099 (2013).

[54] D. A. Fagundes, M. J. Menon, and P. V. R. G. Silva, Nucl. Phys. A946, 194 (2016).

[55] D. A. Fagundes, M. J. Menon, and P. V. R. G. Silva, J. Phys. G 40, 065005 (2013).

[56] A. K. Kohara, E. Ferreira, and T. Kodama, Eur. Phys. J. C 74, 3175 (2014).

[57] I. M. Dremin, JETP Lett. 99, 243 (2014).

[58] I. M. Dremin, Kratk. Soobshch. Fiz. 42, 8 (2015) [Bulletin of the Lebedev Physics Institute 42, 21 (2015)].

[59] I. M. Dremin, Phys. Usp. 58, 61 (2015).

[60] P. Desgrolard, L. L. Jenkovszky, and B. Struminsky, Eur. Phys. J. C 11, 145 (1999).

[61] P. Desgrolard, L. L. Jenkovszky, and B. V. Struminsky, Yad. Fiz. 63, 962 (2000) [Phys. At. Nucl. 63, 891 (2000)]. 